\title{
Stochastic dynamics for a single vibrational mode in molecular junctions
}

\author{
A. Nocera ${ }^{1}$, C.A. Perroni ${ }^{2}$, V. Marigliano Ramaglia ${ }^{2}$ and V. Cataudella ${ }^{2}$ \\ 1 Dipartimento di Fisica E. Amaldi, Universita' di Roma Tre, \\ Via della Vasca Navale 84, I-00146 Roma, Italy \\ 2 CNR-SPIN and Universita' degli Studi di Napoli Federico II \\ Complesso Universitario Monte SantAngelo, Via Cintia, I-80126 Napoli, Italy.
}

\begin{abstract}
We propose a very accurate computational scheme for the dynamics of a classical oscillator coupled to a molecular junction driven by a finite bias, including the finite mass effect. We focus on two minimal models for the molecular junction: Anderson-Holstein (AH) and two-site Su-Schrieffer-Heeger (SSH) models. As concerns the oscillator dynamics, we are able to recover a Langevin equation confirming what found by other authors with different approaches and assessing that quantum effects come from the electronic subsystem only. Solving numerically the stochastic equation, we study the position and velocity distribution probabilities of the oscillator and the electronic transport properties at arbitrary values of electron-oscillator interaction, gate and bias voltages. The range of validity of the adiabatic approximation is established in a systematic way by analyzing the behaviour of the kinetic energy of the oscillator. Due to the dynamical fluctuations, at intermediate bias voltages, the velocity distributions deviate from a gaussian shape and the average kinetic energy shows a non monotonic behaviour. In this same regime of parameters, the dynamical effects favour the conduction far from electronic resonances where small currents are observed in the infinite mass approximation. These effects are enhanced in the two-site SSH model due to the presence of the intermolecular hopping $t$. Remarkably, for sufficiently large hopping with respect to tunneling on the molecule, small interaction strengths and at intermediate bias (non gaussian regime), we point out a correspondence between the minima of the kinetic energy and the maxima of the dynamical conductance.
\end{abstract}

\section{INTRODUCTION}

In recent years it has become possible to fabricate electronic devices where the effective element of a junction is a single molecule placed between two metallic (or semiconductor) electrodes. $\underline{1}^{-3}$ Due to the small size of molecules, the charging of the molecular bridge is often accompanied by significant changes of the nuclear geometry, indicating a strong coupling between electronic and nuclear (in particular vibrational) degrees of freedom. For example, some authors ${ }^{4}$ have recently proposed a theoretical explanation of the switching mechanism, actually observed in different molecular junctions,,$\frac{5}{,}$ based on the electron-phonon interaction. Understanding and controlling the effect of this interaction onto the electric current through molecular devices is not only important for the field of molecular electronics but establishes a strong link also to the physics of Nano-ElectroMechanical Systems (NEMS) 6.7 Recent experiments show the possibility to use single electron transistors coupled to a mechanical oscillator as high sensitive position ${ }^{8,9}$ and mass ${ }^{10}$ sensors. One of the challenges is to understand, control and use the interplay between a quantum ' 'detector' ' (electron transistor) and a classical mechanical system.

The simplest molecular conduction junction comprises two metallic electrodes connected by a single molecule. Such a junction, including the effect of electron-phonon interaction, can be described by the Anderson-Holstein (AH) model. ${ }^{11}$ The molecule is represented by one electronic level interacting linearly with a local vibrational degree of freedom and connected through tunneling with free-electron metals. Electron transport within this model has received a lot of theoretical attention. 12,13 Despite the conceptual simplicity, it gives rise to a very rich physics. Several approaches have been adopted depending on the relative energy scales in the problem. When the characteristic frequency of the oscillator $\omega_{0}$ is of the same order of magnitude of the tunneling frequency of the electrons on the molecule $(\sim \Gamma)$, a quantum treatment of oscillator dynamics is necessary. In this case, it is useful to consider separately the limits of weak and strong electron-phonon interaction strength relative to the coupling of the level to the leads. The former corresponds to nonresonant phonon-assisted electron tunneling, mostly encountered in experiments in inelastic electron tunneling spectroscopy ${ }^{14}$ (IETS), and theoretically understood within Non Equilibrium Green Function (NEGF) formalism. ${ }^{15}-18$ In the case of stronger effective electron-phonon coupling, the perturbative treatment breaks down, the conduction shows phononic blockade at small bias (Franck-Condon effect ${ }^{19-21}$ ) and one can observe phonon sidebands in the conductance spectra. ${ }^{22}$

The interpolation from weak to strong electron-phonon coupling regime in a full quantum description of electron and phonon subsystems is a very challenging problem. However, when the vibrational motion is slow compared to the electronic tunneling rate $\left(\omega_{0}<<\Gamma\right)$, one can apply a simplified scheme in the spirit of Born-Oppenheimer approximation. Indeed, in the zero-order static theory (adiabatic ratio $\omega_{0} / \Gamma \mapsto 0$, oscillator mass $m \mapsto \infty$ ), one neglects the kinetic energy of the oscillator and obtains an exact electronic problem where the oscillator position enters as a parameter to be determined self-consistently. Some authors ${ }^{23-25}$ have already considered the possibility to construct corrections to this picture, within the $\mathrm{AH}$ 
model, in different parameter regimes and with different techniques. Using the action functional formalism on the Keldysh contour for the full interacting electron-phonon

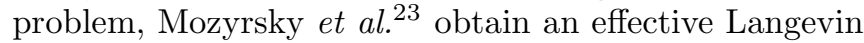
equation for the oscillator field in the limit where the electron-leads are considered as zero temperature thermostats. It comprises a position-dependent dissipation term and white noise force. In the strong electronoscillator coupling regime, where the model shows bistability, they find that the oscillator field acquires an effective temperature linearly related to the bias, $V_{\text {bias }}$, as a consequence of the coupling to the electronic bath.

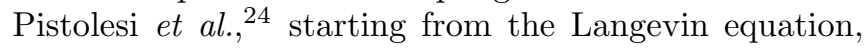
generalize the previous results solving numerically the Fokker-Plank equation associated with it, focusing again on the extremely strong coupling regime. They obtain the dependence of the current on the transport and gate voltages, as well as address the problem of mechanical switching between the metastable states of the oscillator potential.

A similar approach, based on the Feynman-Vernon influence functional, was recently adopted by Hussein et $a l . \stackrel{25}{n}$ obtaining the same Langevin equation. However, they do not solve this equation and study the phase space portraits of the Newton equation obtained in absence of electronic bath induced noise. Moreover, they extend this analysis to a molecular double dot molecular Hamiltonian. For both cases they have explained the features of effective potential and friction terms entering the oscillator equation of motion.

The approaches discussed above, based on an expansion of the action in the adiabatic limit, are not able to disentangle the origin of the quantum effects in the Langevin equation for the oscillator. We propose here an alternative and more direct way that allows us to clarify this point.

We construct systematically the dynamical finite mass corrections to the static theory and their influence on the transport problem in the following way: we perform an adiabatic expansion on the electron-oscillator self-energy following the approach of Ref.26, obtaining a corresponding expansion of the Green function. This expansion gives rise to the same friction and fluctuating terms obtained with action functional techniques, but clarifies that the quantum effects 'hidden' in the stochastic equation come only from the electronic subsystem.

We numerically solve the Langevin equation deriving the position and velocity distribution probabilities of the oscillator for a very large range of the relevant parameters. We find that, at intermediate bias voltages, the velocity distributions $P(v)$ deviate from a gaussian shape as a result of the coupling of the oscillator with the outof-equilibrium electronic bath ${ }^{27}$ Correspondingly, the kinetic energy of the oscillator shows a non monotonic behaviour as function of the bias due to the slight change of the force exerted on the oscillator.

We study transport properties like the current-voltage characteristic and the conductance, observing in the $\mathrm{AH}$ model a dynamical reduction of the the 'polaronic' shift and the broadening of the electronic resonance due to the average over the nonequilibrium position distribution probability of the oscillator. We note an interesting strong enhancement of current in the non gaussian region at intermediate bias, where the infinite mass approximation prescribes very small conduction.

It is of paramount importance to study the range of validity of the adiabatic approach. Making a thorough investigation of this issue is crucial in order to get a match with experimental results and exact theoretical calculations. We establish the range of validity of the adiabatic approximation analyzing systematically the behaviour of the oscillator's kinetic energy. We are able to build up a diagram for the validity of classical approximation, identifying Quantum (QR), Classical-Adiabatic (CAR) and Classical Non-Adiabatic (CNAR) Regions. We compare the classical kinetic energy of the oscillator with the Debye temperature $\left(k_{B} T_{D} \sim \hbar \omega_{0}\right)$ to distinguish between $\mathrm{QR}$ against CAR regimes, and with electron energy scale $(\sim \hbar \Gamma)$ to distinguish between CAR against CNAR regimes.

We extend this analysis to the case of a molecular Hamiltonian composed by a couple of sites interacting with a single vibrational mode in the $\mathrm{SSH}$ model ${ }^{28-32} \mathrm{In}$ this case, because of the direct coupling of the electronoscillator interaction to the intermolecular hopping, one expects that the role of the dynamical fluctuations becomes crucial to determine the correct features of the observables inherent to the transport problem.

In the limit of symmetric coupling with leads, we are able to construct again a Langevin equation for the oscillator dynamics, very similar to that derived in $\mathrm{AH}$ model. In this case, it is possible to study the effect of the dynamics of the classical vibrational mode on the electron hopping through the two molecular sites. Vice-versa, one can also study the effect of intermolecular electron degree of coherence onto the vibrational dynamics.

The new intermolecular electronic hopping scale $t$ introduces a reduction of the CAR in the validity diagram. This reduction becomes important if $t / \hbar \Gamma>1$ with the occurrence of QRs. These new features are due to the stronger nonmonotonic behaviour of the kinetic energy of the oscillator as function of the bias voltage. For sufficiently large $t / \hbar \Gamma$, small interaction strengths and at intermediate bias (non gaussian regime), the kinetic energy curves show well defined minima. We point out a correspondence between these minima and the maxima of the conductance. Again, as concerns the electronic transport properties, the dynamical fluctuations favour the conduction far from the two electronic (static) resonances. In the SSH model, as already stressed above, the effects of the dynamical fluctuations become even more important. One can observe again a complete erasing of the bistability and hysteretic behaviour predicted by the infinite mass approximation.

The paper is organized as follows: In Sec. II we present the single level case within the AH model. In Sec. III we 
deal with the case of two-site-SSH model.

\section{THE ANDERSON-HOLSTEIN (AH) MODEL}

The spinless Anderson-Holstein model is the simplest model of a molecular junction including the effect of electron-phonon interaction. The molecule is modeled as a single electronic level interacting locally with a single vibrational mode. The electronic system is described by the standard junction Hamiltonian $\hat{\mathcal{H}}_{e l}=$ $\hat{H}_{\text {mol }}+\hat{H}_{\text {tun }}+\hat{H}_{\text {leads }}$, with

$$
\begin{gathered}
\hat{H}_{m o l}=E_{g} \hat{d}^{\dagger} \hat{d}, \\
\hat{H}_{\text {tun }}=\sum_{k, \alpha}\left(V_{k, \alpha} \hat{c}_{k, \alpha}^{\dagger} \hat{d}+\text { h.c. }\right), \\
\hat{H}_{\text {leads }}=\sum_{k, \alpha} \varepsilon_{k, \alpha} \hat{c}_{k, \alpha}^{\dagger} \hat{c}_{k, \alpha} .
\end{gathered}
$$

The molecular electronic level has energy $E_{g}$ and creation (annihilation) operators $\hat{d}^{\dagger}(\hat{d})$. The operators $\hat{c}_{k, \alpha}^{\dagger}\left(\hat{c}_{k, \alpha}\right)$ create (annihilate) electrons with momentum $k$ and energy $\varepsilon_{k, \alpha}=\xi_{k, \alpha}-\mu_{\alpha}$ in the left $(\alpha=L)$ or right $(\alpha=R)$ free metallic leads. The chemical potentials in the leads, $\mu_{L}$ and $\mu_{R}$, are assumed to be biased by an external voltage, $e V_{\text {bias }}=\mu_{L}-\mu_{R}$. Electronic tunneling between the molecular dot and a state in the lead has amplitude $V_{k, \alpha}$. We consider the oscillator dynamics 'classical' from the beginning and described by the position and momentum variables $x, p$.

The Hamiltonian of the oscillator is given by

$$
H_{o s c}=\frac{p^{2}}{2 m}+\frac{1}{2} m \omega_{0}^{2} x^{2},
$$

characterized by the frequency $\omega_{0}$ and the effective mass $m$. The interaction (typically of electrostatic origin) is provided by a simple linear coupling between the electron occupation on the molecule, $\hat{d}^{\dagger} \hat{d}$, and the displacement of the oscillator

$$
\hat{H}_{\text {int }}=\lambda x \hat{d}^{\dagger} \hat{d},
$$

where $\lambda$ is the electron-oscillator coupling (EOC) strength. The overall Hamiltonian is therefore given by $\hat{\mathcal{H}}=\hat{\mathcal{H}}_{\text {el }}+H_{\text {osc }}+\hat{H}_{\text {int }}$.

In the following, the coupling between the electron system and the vibrational mode will be often described in terms of the electron-phonon coupling energy $E_{p}=$ $\lambda^{2} /\left(2 m \omega_{0}^{2}\right)$, while the coupling to the leads by the tunneling rate $\Gamma_{k, \alpha}=2 \pi \rho_{\alpha}\left|V_{k, \alpha}\right|^{2} / \hbar$ (the full hybridization width of the molecular orbital is then $\hbar \Gamma_{k}=\hbar \Gamma_{k, L}+$ $\hbar \Gamma_{k, R}$ ), where $\rho_{\alpha}$ is the density of states of the lead $\alpha$. For the sake of simplicity, we will suppose flat density of states for the leads within the wide-band approximation
$\left(\Gamma_{k, \alpha} \mapsto \Gamma_{\alpha}\right)$. In this paper we will measure length in units of $x_{0}=\frac{\lambda}{m \omega_{0}^{2}}$ and energy in units of $\hbar \Gamma$. Finally, the leads will be considered as zero temperature thermostats.

In the next subsections, we will first (subsection A) analyze the coupled electron-oscillator problem in the limit of infinite mass for the oscillator. We will then indicate how to construct (subsection B) the stochastic Langevin equation for the dynamics of the oscillator including the finite mass effect. In the subsection $\mathrm{C}$ we will solve numerically the stochastic equation and analyze the effects of the oscillator dynamics on the electronic observables inherent to the transport problem (I-V characteristic and conductance).

\section{A. Out of equilibrium Born-Oppenheimer approximation: infinite mass (static) case}

When the vibrational motion of the oscillator is slow with respect to all electronic energy scales, it is possible to decouple oscillator and electronic dynamics. In the spirit of Born-Oppenheimer approximation, we consider the limit $m \mapsto \infty$ in the full Hamiltonian disregarding the kinetic energy of the oscillator. The electronic dynamics is therefore equivalent to a non-interacting resonant single level problem with energy level renormalized by the 'polaronic' shift $E_{g} \mapsto E_{g}+\lambda x$. The retarded (advanced) Green functions $G^{r(a)}(\omega, x)$ and the lesser (greater) Green functions $G^{<(>)}(\omega, x)$ in stationary nonequilibrium conditions are derived within the Keldysh formalism 16,17 and depend parametrically by the displacement coordinate $x$. Starting from the force exerted on the oscillator

$$
F=-m \omega_{0}^{2} x+\lambda\left\langle\hat{n}_{e l}\right\rangle(x),
$$

where

$$
\left\langle\hat{n}_{e l}\right\rangle(x)=\sum_{\alpha=L, R} \hbar^{2} \Gamma_{\alpha} \int \frac{d \omega}{2 \pi} f_{\alpha}(\omega)\left|G^{r}(\omega, x)\right|^{2},
$$

with $f_{\alpha}(\omega)$ Fermi function of the lead $\alpha=R, L$, one can therefore straightforwardly compute the expression of the generalized potential in nonequilibrium conditions (obtained applying symmetric bias unbalance $\mu_{R}=$ $\left.-e V_{\text {bias }} / 2, \mu_{L}=e V_{\text {bias }} / 2\right)$

$$
\begin{aligned}
& U(x)=\frac{1}{2} m \omega_{0}^{2} x^{2}+\frac{\lambda x}{2}-\sum_{\alpha=L, R}\left[\frac{\mu_{\alpha}-E_{g}-\lambda x}{2 \pi} \times\right. \\
& \arctan \left(\frac{\mu_{\alpha}-E_{g}-\lambda x}{\hbar \Gamma / 2}\right)-\frac{\hbar \Gamma}{8 \pi} \ln \left[4\left(\mu_{\alpha}-E_{g}-\lambda x\right)^{2}\right. \\
& \left.\left.+(\hbar \Gamma)^{2}\right]\right] .
\end{aligned}
$$

This generalized oscillator potential depends parametrically by the spring constant $m \omega_{0}^{2}=k$, the EOC strength $\lambda$, the energy of the electron level $E_{g}$ (which 


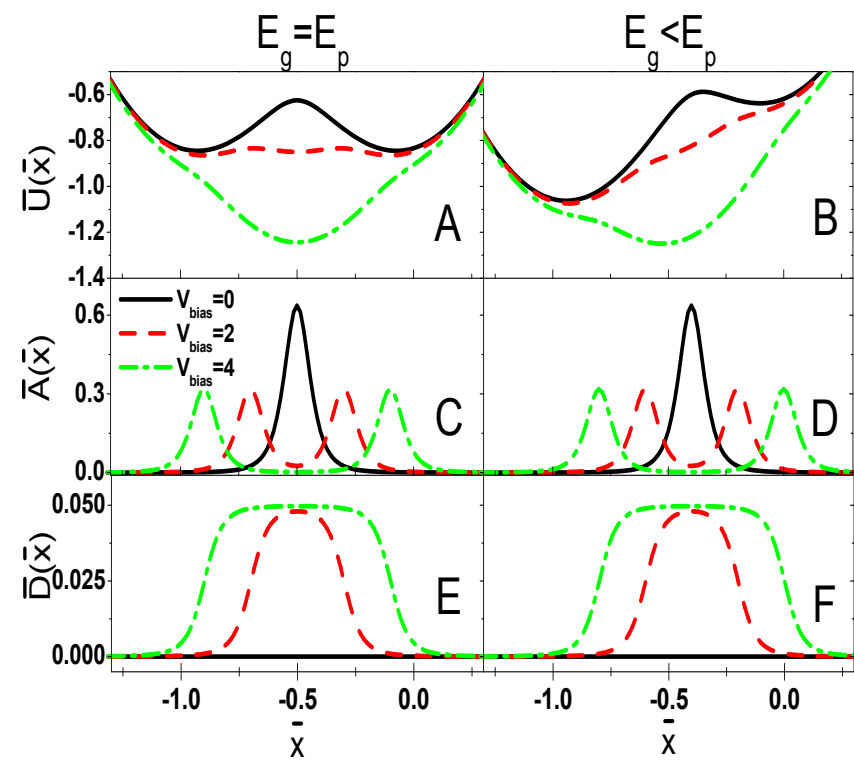

FIG. 1. (Color online). Spatial dependence of the dimensionless generalized static potential $\bar{U}(\bar{x})$ (panels A,B), friction coefficient $\bar{A}(\bar{x})$ (panels C,D), fluctuating term $\bar{D}(\bar{x})$ (panels E,F) for symmetric $E_{g} \sim E_{p}$ and asymmetric $E_{g}<E_{p}$ minima and different values of bias, $V_{\text {bias }}=0$ (solid (black) curve), $V_{\text {bias }}=2$ (dashed (red) curve), $V_{\text {bias }}=4$ (dashed dot (green) curve). The potential is expressed in $\hbar \Gamma$ units $(\bar{U}=$ $U / \hbar \Gamma)$, the friction coefficient in $m \omega_{0}$ units $\left(\bar{A}=A / m \omega_{0}\right)$, the fluctuating term in $\lambda^{2} / \omega_{0}$ units, $\left(\bar{D}=D /\left(\lambda^{2} / \omega_{0}\right)\right)$. $V_{\text {bias }}$ values are expressed in $\hbar \Gamma / e$ units, where $e$ is the electron charge. The dimensionless position variable $\bar{x}$ is defined as $\bar{x}=x / x_{0}$ with $x_{0}=\frac{\lambda}{m \omega_{0}^{2}}$.

can be considered a gate potential), the coupling to the leads $\Gamma$ and finally by the bias, $V_{\text {bias }}$. In Fig 1 (panels $\mathrm{A}, \mathrm{B})$, we present some features of the generalized potential $U(x)$ in the strong coupling regime $\left(E_{p}>\hbar \Gamma\right)$, where the potential shows several minima. For $E_{g} \sim E_{p}$ and not too large bias (panel A, Fig,1), the potential develops two symmetric minima near $x \simeq 0$ (corresponding to $\left.\left\langle\hat{n}_{e l}\right\rangle \simeq 0\right)$ and $x \simeq-1$ (corresponding to $\left\langle\hat{n}_{e l}\right\rangle \simeq 1$ ) separated by a barrier whose height is roughly proportional to $E_{p}$. This bistable regime corresponds to the physical situation where the bare electron level $E_{g}$ is above the chemical potential of both leads, while the renormalized charged level $E_{g}-2 E_{p}$ is below them. The molecule can stay in one minimum or in the other. If we increase the bias $V_{\text {bias }}$, the potential $U(x)$ shows a third minimum corresponding to average electron occupation on the molecule $\left\langle\hat{n}_{e l}\right\rangle \simeq 1 / 2$ and, for sufficiently large $V_{\text {bias }}$, only this minimum remains. If $E_{g}<E_{p}$ the potential also shows two or more minima but they are asymmetric (panel B, Fig 1). For sufficiently large bias, the common feature is the existence of a single minimum corresponding to occupation $\left\langle\hat{n}_{e l}\right\rangle \simeq 1 / 2$.

In the above analysis the displacement $x$ has been used as a free parameter. Actually, the only $x$ values relevant for the electronic properties in the static approximation are those which solve the equation $F(x)=0$. These solutions depend parametrically by all the parameters of the theory (in particular by the bias $V_{\text {bias }}$ ). This may yield transitions between different local minima in the potential, determining in the electronic current-voltage characteristic the onset of interesting non linear phenomena like hysteresis, bistability and Negative Differential Re-

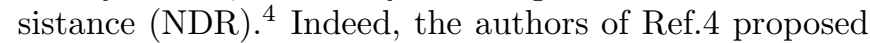
a polaron mechanism within the $\mathrm{AH}$ model to explain such phenomena, effectively observed in transport experiments on molecular devices. However, the results of the static approximation can be strongly modified by dynamical effects. Indeed, corrections due to the finite (though large) mass of the oscillator are expected to be important $2 \underline{23}-\underline{25}$ As we shall see in the next sections, the inclusion of the finite mass effect on the oscillator dynamics gives rise to a stochastic Langevin equation with a position dependent dissipation term and white noise force. The stochastic fluctuations of the oscillator motion will strongly modify the current-voltage characteristics obtained in the infinite mass approximation.

\section{B. Dynamical (finite mass) corrections to static case: setting Langevin equation for the oscillator}

Within the static approximation (infinite mass), the main effect of the nonequilibrium fast electronic environment is the modification of the force (Eq. ([6]) ) experienced by the mechanical oscillator. The oscillator has no dynamics at all and the displacements $x$ are 'frozen' in suitable points of the configuration space given by the equation $F(x)=0$. In this section, we show how to include dynamical corrections due to finite mass of the oscillator.

First of all, we should include the time dependence of the oscillator dynamics in the Hamiltonian of the electronic problem (where we still neglect the oscillator kinetic energy, $\hat{\mathcal{H}} \mapsto \hat{\mathcal{H}}(x(t)))$. Using the extension of the Keldysh formalism to time dependent cases, $\underline{17}$ we can solve the Dyson and Keldysh equations for the molecular Green functions which now depends on times $t$ and $t^{\prime}$ separately. The retarded molecular Green function can be obtained analitically (with $E_{g}(t)=E_{g}+\lambda x(t)$ )

$$
G^{r}\left(t, t^{\prime}\right)=-\frac{\imath}{\hbar} \theta\left(t-t^{\prime}\right) e^{-\imath \int_{t^{\prime}}^{t} d t_{1}\left(\frac{E g\left(t_{1}\right)}{\hbar}-\imath \Gamma / 2\right)},
$$

and depends in non linear way by the entire dynamics $x(t)$ of the oscillator. Indeed, the dependence by the entire history of the oscillator motion prevents us from Fourier transforming the Green function and leads to an intractable problem.

In order to overcome this difficulty, we resort to an adiabatic approximation of the molecular Green function. As thoroughly discussed in the Appendix $A$, one should restart from the Dyson equation for the Green function (Eq. (A1)) and perform an expansion in the 
electron-phonon self-energy where a separation between 'slow' and 'fast' time scales was preliminary accomplished (Eqs. (A3|A4)). The expansion, performed with respect to the 'slow' time $\left(t+t^{\prime}\right) / 2$, allows to disentangle the non-local time dependence of the Green function. In the end, the truncated Green function can be Fourier transformed with respect to 'fast' time $t-t^{\prime}$, gaining a 'slow' time dependence and a linear correction in the oscillator velocity (See Eqs. A8|A9|A10).

\section{Abiabatic Approximation: calculation of damping and fluctuating term}

We have now the tools to calculate the adiabatic corrections to the force acting on the mechanical oscillator. For the sake of clarity, we rewrite here the adiabatic expansion of the Fourier transformed molecular Green function derived in the Appendix A (Eq A8)

$$
G^{r}(\omega, t) \simeq G_{0}^{r}(\omega, t)+G_{1}^{r}(\omega, t) .
$$

The explicit expressions of $G_{0}^{r}$ and $G_{1}^{r}$ are given by Eqs. A9A10. Actually, in order to introduce dynamical effects on the force Eq.(6), we have to calculate the adiabatic corrections to the lesser-Green function that is directly related to the occupation

$$
\begin{aligned}
& \langle\hat{n}\rangle(t)=-\imath \hbar G^{<}(t, t)= \\
& \sum_{\alpha=L, R} \hbar^{2} \Gamma_{\alpha} \int \frac{d \omega}{2 \pi} f_{\alpha}(\omega)\left|G^{r}(\omega, t)\right|^{2} .
\end{aligned}
$$

From Eq. (A9), one obtains, at zero-order, an expression for the occupation of the same form of the static limit (Eq.(7)) with the substitution $E_{g} \leftrightarrow E_{g}(t)$, acquiring a weak time dependence through the slow variable $t$. Adding the first order correction Eq. A10 into the Eq.(11), and neglecting terms proportional to the square velocity of the oscillator, we obtain

$$
\begin{aligned}
& \langle\hat{n}\rangle(t) \simeq \sum_{\alpha=L, R} \hbar^{2} \Gamma_{\alpha} \int \frac{d \omega}{2 \pi} f_{\alpha}(\omega)(1+ \\
& \left.\frac{\hbar \Gamma}{2} \frac{\partial E_{g}}{\partial t}\left|G_{0}^{r}(\omega, t)\right|^{2} \frac{\partial}{\partial \omega}\right)\left|G_{0}^{r}(\omega, t)\right|^{2} .
\end{aligned}
$$

In the end, the force given by Eq.(6) modifies to

$$
F(x) \mapsto F^{\prime}(x, v)=F(x)-A(x) v,
$$

where $v=\dot{x}$ is the velocity of the oscillator. We can conclude that, both in equilibrium and in out-of-equilibrium conditions, the interaction with the leads introduces a dissipative correction term to the oscillator dynamics.

Now, we observe that the introduction of a dissipative term cannot be the unique dynamical effect for a classical dynamical system in contact with an environment. It is well known that a fluctuating term should be included to take correctly into account the effect of the bath degree's of freedom. In our case, in order to include completely the effect of the 'fast' electronic environment on the oscillator motion, we propose to take into account the fluctuations of the force ${ }^{33}$ acting on the oscillator. These are induced by the intrisic 'quantum' fluctuations of the electronic subsystem.

We add to the average force contribution Eq.(6), suitable corrected by the damping term, Eq.(13), a stochastic fluctuating term able to take into account the effect of the electronic quantum fluctuations on the classical dynamics of the oscillator. Indeed, because we are considering zero-temperature leads, one expects that these fluctuations are triggered by a finite bias voltage applied to the junction. As we shall see in the next sections, our approach is very accurate for junctions driven by a finite bias voltages, but it is far less accurate to describe the physics in the small bias (quantum) regime.

We estimate the noise strength evaluating the average of the square fluctuation of the force over the electronic steady state. This fluctuating term is directly related to the fluctuation of the electron occupation

$$
\begin{aligned}
& \left\langle\delta \hat{F}(t) \delta \hat{F}\left(t^{\prime}\right)\right\rangle=\lambda^{2}\left\langle\delta \hat{n}(t) \delta \hat{n}\left(t^{\prime}\right)\right\rangle= \\
& \lambda^{2}\left(\left\langle\hat{n}(t) \hat{n}\left(t^{\prime}\right)\right\rangle-\langle\hat{n}(t)\rangle\left\langle\hat{n}\left(t^{\prime}\right)\right\rangle\right) .
\end{aligned}
$$

Decoupling the term $\left\langle\hat{n}(t) \hat{n}\left(t^{\prime}\right)\right\rangle$ with the Wick theorem, one obtains

$$
\begin{aligned}
& \left\langle\delta \hat{F}(t) \delta \hat{F}\left(t^{\prime}\right)\right\rangle=\lambda^{2} \hbar^{2} G^{<}\left(t^{\prime}-t\right) G^{>}\left(t-t^{\prime}\right)= \\
= & \lambda^{2} \hbar^{2} G_{0}^{<}\left(t^{\prime}-t\right) G_{0}^{>}\left(t-t^{\prime}\right),
\end{aligned}
$$

where we have used zero-order time-dependent Green functions (as in Eq. (A9)) in order to take only first order corrections in the adiabatic ratio $\frac{\omega_{0}}{\Gamma}$. At this level of approximation, we have obtained a multiplicative coloured noise $\stackrel{34}{=}$ According to the adiabatic approximation, we can further simplify the fluctuating term retaining only the zero-frequency component of the noise

$$
\begin{aligned}
& \lim _{\varepsilon \mapsto 0} \lambda^{2} \hbar^{2} \int d \varepsilon e^{\imath \varepsilon\left(t-t^{\prime}\right)} \int \frac{d \omega}{2 \pi} G_{0}^{<}(\omega+\varepsilon) G_{0}^{>}(\omega) \\
& \simeq D(x) \delta\left(t-t^{\prime}\right),
\end{aligned}
$$

corresponding to electronic times scales comparable with that of the oscillator. In this way, one obtains a multiplicative white noise term in the equation of motion for the oscillator $\stackrel{34}{\underline{3}}$ The resulting Langevin equation for the oscillator dynamics becomes

$$
\begin{gathered}
m \ddot{x}+A(x) \dot{x}=F(x)+\sqrt{D(x)} \xi(t), \\
\langle\xi(t)\rangle=0, \quad\left\langle\xi(t) \xi\left(t^{\prime}\right)\right\rangle=\delta\left(t-t^{\prime}\right),
\end{gathered}
$$

where $\xi(t)$ is a standard white noise term. Explicitly, the damping term $A(x)$ is given by (from Eq.(12))

$$
A(x)=\frac{4 m \omega_{0}}{\pi} \frac{\hbar \omega_{0}}{\hbar \Gamma} \frac{E_{p}}{\hbar \Gamma} \sum_{\alpha=L,-R}\left(\frac{1}{\left[\left(\frac{\mu_{\alpha}-E_{g}-\lambda x}{\hbar \Gamma}\right)^{2}+1\right]^{2}}\right),
$$


while the fluctuating term is (from Eq.(16))

$$
\begin{aligned}
D(x) & =\frac{m \omega_{0} E_{p}}{\pi} \frac{\hbar \omega_{0}}{\hbar \Gamma} \sum_{\alpha=L,-R}\left(\arctan \left(\frac{\mu_{\alpha}-E_{g}-\lambda x}{\hbar \Gamma}\right)\right. \\
& \left.+\frac{\frac{\mu_{\alpha}-E_{g}-\lambda x}{\hbar \Gamma}}{\left[\left(\frac{\mu_{\alpha}-E_{g}-\lambda x}{\hbar \Gamma}\right)^{2}+1\right]}\right)
\end{aligned}
$$

where it is understood that $\sum_{\alpha=L,-R} K(\alpha)=K(L)-$ $K(R)$, for a generic function $K(\alpha)$. We note that Eqs.(17), (18) and (19) are identical to that obtained in Ref.23 - 25. Introducing a natural temporal unit $t_{0}=1 / \omega_{0}$, the dimensionless damping $\bar{A}(\bar{x})$ and fluctuating $\bar{D}(\bar{x})$ coefficients result proportional to the adiabatic ratio $\omega_{0} / \Gamma$. As concerns the spatial dependence of the damping term, one can note in Fig 1 (panels C-D) that it is almost localized on the position of the local minima of the static potential. The fluctuating coefficient, as shown in Fig 1 (solid (black) line in panels E-F), vanishes at equilibrium (bias voltage $V_{\text {bias }}=0$ ). Only applying finite bias it becomes different from zero. In Fig [1 (dashed (red) and dashed dotted (green) lines in panels E-F), we show that its spatial extension increases as the bias increases. Finally, one can note that $A(x)$ and $D(x)$ are almost independent by the ratio $E_{g} / E_{p}$.

\section{Numerical solution of Langevin equation: electronic observables and limits of the stochastic approach}

From the Langevin equation Eq. (17) it is possible to derive the distribution probabilities $P(x)$ and $P(v)$ for the position and velocities variables of the oscillator. We have evaluated them solving the second order stochastic differential equation with the 4-order stochastic RungeKutta algorithm developed by R. L. Honeycutt. ${ }^{35,36}$ First of all, as suggested in Ref.37, in order to solve our second order ordinary differential equation with multiplicative white noise, we decompose the problem in a set of three first order differential equations. The third one takes into account the effect of spatial dependence of the noise involving a non multiplicative noise term. For our simulations we have fixed a time step $t_{s}=0.1 \tau\left(\tau=1 / \omega_{0}\right)$ and set long simulation times up to $T=10^{9} t_{s}$. Within these settings, the algorithm shows an excellent stability in the whole range of model parameters. In order to construct our histograms, we have sampled the values of $x(t)$ and $v(t)$ every 100 time steps. We have therefore obtained the distribution probabilities for the stationary state of the oscillator.

Given our assumption about the separation between the slow ionic (vibrational) and fast electronic (tunneling) timescales, the problem of evaluating a generic observable (electronic or not) of the system reduces to the evaluation of that quantity for a fixed position $x$ and velocity $v$ of the oscillator, with the consequent averaging over the stationary probability distributions, $P(x)$ and
$P(v)$. Therefore, for a generic observable which depends only on position, $O(x)$, the averaged quantity is

$$
\langle O(x)\rangle=\int d x P(x) O(x),
$$

while, for an observable which depends on velocity variable only, one has

$$
\langle O(v)\rangle=\int d v P(v) O(v) .
$$

In our case, the current, the spectral function and the electronic occupation depend only on the position variable

$$
\begin{aligned}
I(x) & =\frac{e}{\hbar} \int_{\mu_{R}}^{\mu_{L}} \frac{d \hbar \omega}{2 \pi} \frac{\hbar \Gamma_{L} \hbar \Gamma_{R}}{\hbar \Gamma} A_{S p e c}(\omega, x), \\
A_{\text {Spec }}(\omega, x) & =\frac{\hbar \Gamma}{\left(\hbar \omega-E_{g}-\lambda x\right)^{2}+\hbar^{2} \Gamma^{2} / 4}, \\
\langle\hat{n}\rangle(x) & =\frac{1}{2}+\frac{1}{2 \pi} \sum_{\alpha=R, L} \arctan \left[\frac{\mu_{\alpha}-E_{g}-\lambda x}{\hbar \Gamma / 2}\right] .
\end{aligned}
$$

The position distribution probabilities $P(x)$ have been already discussed by authors of Ref.24 in the extremely strong coupling regime $E_{p}>>\hbar \Gamma>>\hbar \omega_{0}$. They analyze the case where the static potential shows two symmetric or asymmetric wells separated by a very high barrier. In this regime, solving numerically the Fokker-Plank equation of the problem, they estimate the switchingrates by evaluating the escape times from each well of the generalized potential. Indeed, this point is interesting for clarifying the role of electron-phonon interaction in the appearance of a bistable behaviour in single molecule tunneling devices. One of the results of this paper is that the multistability and hysteretic behaviour in the current-voltage characteristic disappear if the dynamical effects of the oscillator motion are taken into account. To clarify this point, we focus here on the case (already considered in Ref.24) where the switching times between different oscillator potential wells are very long, and the oscillator jumps between two states (see panel A of Fig 21) corresponding to very small electronic currents. In order to explore the same regime of parameters, in our approach very long simulation times as $T=10^{9} t_{s}$ are necessary for sampling the entire phase space experienced during the dynamics. Nevertheless, as shown in Panel B of Fig, 2, we get an excellent agreement with Pistolesi et al. results. It is interesting to note that in the paper of Ref.24, the authors consider a broadening $\Gamma$ which is twice our values (we show in the caption of Fig 2 the comparison between the simulations taking correctly into account this factor). In the small bias regime, we observe a strong suppression of the current. The oscillator spends a long time in each potential well, suddenly jumps into the other and then comes back in the same way (see panel A in Fig 2). For clarity, we show in panel C of Fig 2 the corresponding position distribution probability $P(x)$. 


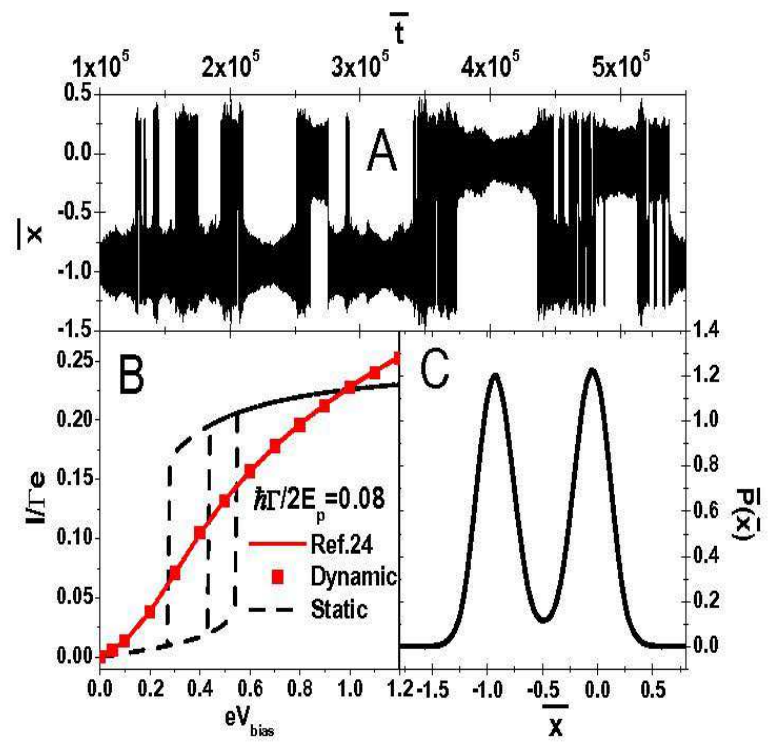

FIG. 2. (Color online). Panel A: Solution of the Langevin equation Eq. (17) in the extremely strong coupling regime $E_{p}>>\hbar \Gamma>>\hbar \omega_{0}\left(\hbar \omega_{0} / 2 E_{p}=10^{-3}\right)$ for $\hbar \Gamma / 2 E_{p}=0.08$, $E_{g}=E_{p}$ and $e V_{\text {Bias }} / 2 E_{p}=0.1$. Panel B: Current ( $e \Gamma$ units) voltage ( $e V_{\text {bias }}$ in $2 E_{p}$ units) characteristic for the same value of $\hbar \Gamma / 2 E_{p}$ as above. Solid (red) curve is drown from Ref.24, square line indicates our dynamic simulation and dashed line indicates static I-V. Panel C: Dimensionless position distribution probability for the same values of parameters as in Panel A. The dimensionless position $\bar{x}$, time $\bar{t}$ and distribution function $\bar{P}$ are defined as $\bar{x}=x / x_{0}, \bar{t}=t / t_{0}, \bar{P}=P /\left(1 / x_{0}\right)$, with $x_{0}=\frac{\lambda}{m \omega_{0}^{2}}$ and $t_{0}=1 / \omega_{0}$, respectively.

The maxima of $P(x)$ correspond to two small current carrying states: the position of the molecular energy level is far above $\left(E_{g} \sim E_{p}\right)$ or below $\left(E_{g} \sim-E_{p}\right)$ the chemical potential of the leads. For sufficiently large bias voltage, as discussed in subsection $\mathrm{A}$, appears a third minimum in the static potential. This minimum corresponds to a large-current carrying state determining a continuous enhancement of the current, against the abrupt discontinuity (or hysteresis) which would been obtained in the static approximation (dashed line in panel B of Fig 22).

\section{Non gaussian features of $P(v)$ and study of the average kinetic energy of the oscillator}

In this section we focus our attention on the oscillator observables $O(v)$ which depend on the velocity $v$. We remark that the oscillator is coupled to the electronic bath only through the interaction term $\hat{H}_{i n t}$, Eq.(15). As the bias voltage increases, this bath is strongly driven out of equilibrium. It is therefore important to analyze the effect of the electronic subsystem on the oscillator distribution probability $P(v)$ as a function of the bias voltage. In the small bias regime, regardless the value of the
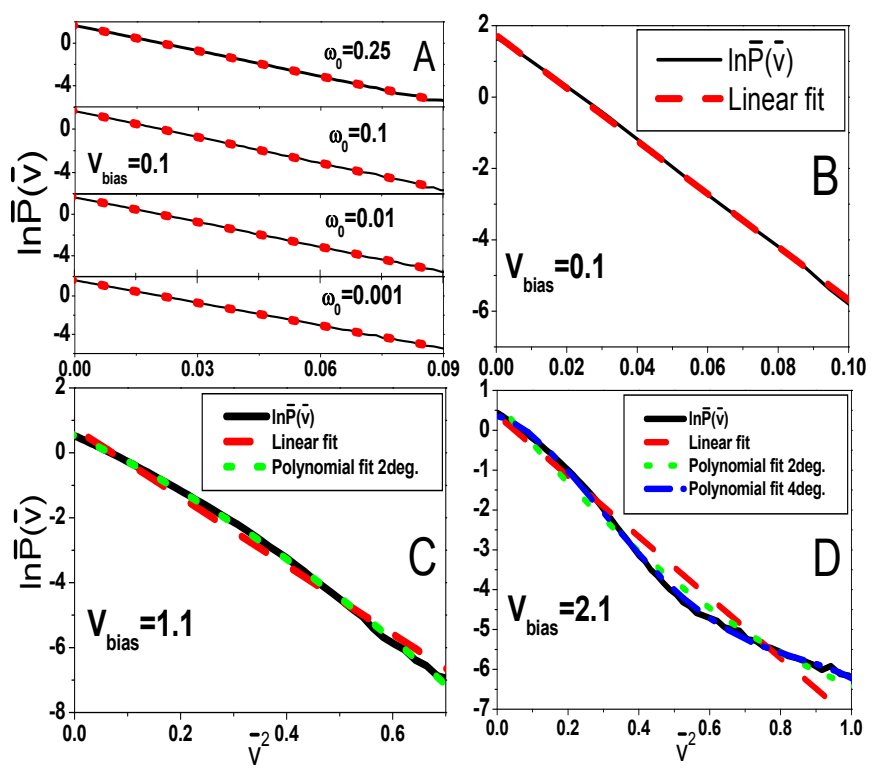

FIG. 3. (Color online). Panel A: log-plot of dimensionless velocity probability distribution function vs. $v^{2}$, at different adiabatic ratios (the values of $\omega_{0}$ shown in the figure are in $\Gamma$ units), fixed bias voltage $V_{\text {bias }}=0.1$ and different gate voltages and EOC strengths (not shown in the graph). The dotted (red) lines indicate that curves have a good linear fit. Panels $B-C-D$ : log-plot of velocity probability distribution function vs. $v^{2}$ for $V_{\text {bias }}=0.1, V_{\text {bias }}=1.1, V_{\text {bias }}=2.1$, respectively. The dashed (red) line indicates linear fitting. Dotted (green) and dash dotted (blue) lines indicate polynomial fitting of 2 nd and 4 th degree. $V_{\text {bias }}$ values are expressed in $\hbar \Gamma / e$ units. The dimensionless distribution function is defined as $\bar{P}=P /\left(m \omega_{0} / \lambda\right)$, while $v^{2}$ is expressed in $\left(\lambda / m \omega_{0}\right)^{2}$ units.

gate voltages $E_{g}$ and the coupling $E_{p}$, as shown in Fig 3 (panel $A$ ) for different adiabatic ratios (from $\omega_{0}=10^{-3}$ to $\left.\omega_{0}=0.25\right)$, the velocity distribution probabilities $P(v)$ are gaussian. In this regime, the nonequilibrium electronic bath behaves like a conventional bath for the oscillator with an 'effective' temperature linearly proportional to the bias voltage. As described in the inset of Fig 4, at arbitrary $E_{p}$ and gate voltages the kinetic energy curves show a common linear trend at small bias with a slope $V_{\text {bias }} / 4$ in agreement with Mozyrsky et al. (we get $V_{\text {bias }} / 8$ because we choose a broadening $\hbar \Gamma$ half that used in Ref.23). As we increase the bias voltage, the $\left(\log P(v)\right.$ vs. $\left.v^{2}\right)$ plot starts to deviate from a linear trend, as shown in Fig 3 , panels $B-C-D$. This behaviour indicates that the oscillator dynamics cannot be simply reduced to an effective temperature in this regime, pointing to a very significant role of the dynamical effects.

In the adiabatic approximation, the average kinetic energy of the oscillator has an important role. It describes the effect of the 'back-action' of the nonequilibrium electronic bath on the oscillator dynamics and can be used, as shown below, as a tool to assess the validity of the adiabatic approximation. We show in Fig 4 the behaviour of the kinetic energy $\left\langle E_{\text {Kin }}\right\rangle$ for dif- 


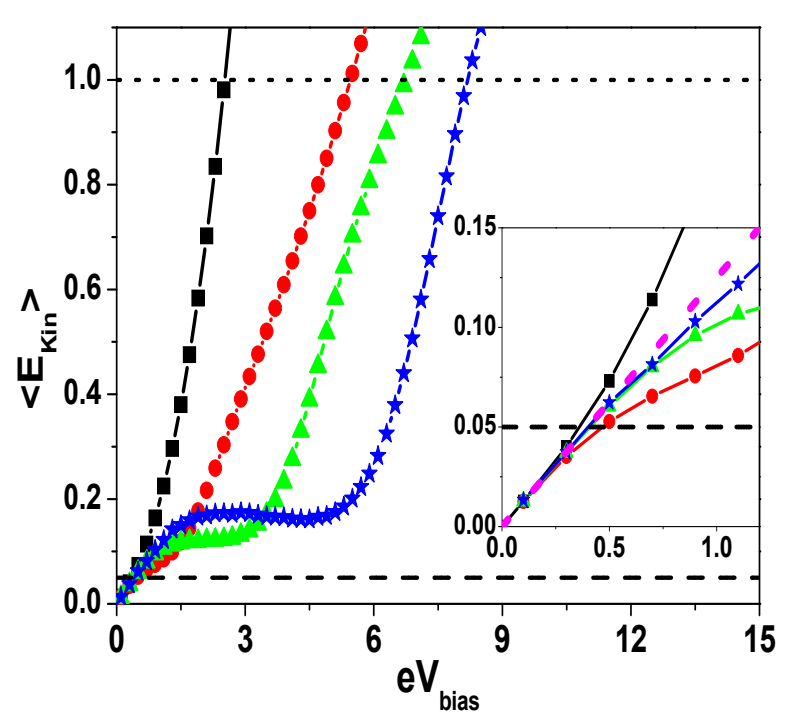

FIG. 4. (Color online). Main: plot of average kinetic energy $\left\langle E_{K i n}\right\rangle$ as function of the bias voltage at fixed adiabatic ratio $\omega_{0} / \Gamma=0.1$ and gate voltage $E_{g}=0$, for different interaction strengths $E_{p}: E_{p}=0.1$ square (black) curve, $E_{p}=1.0$ circle (red) curve, $E_{p}=2.0$ triangle (green) curve, $E_{p}=3.0$ star (blue) curve. Two constant energy lines $E=\hbar \omega_{0} / 2 \hbar \Gamma=$ 0.05 (dashed) and $E=\hbar \Gamma=1$ (dotted) are also plotted. Inset: Average kinetic energy $\left\langle E_{K i n}\right\rangle$ for small bias voltages for the same parameter values of the main plot. The dotted (magenta) line indicates the linear approximation $e V_{\text {bias }} / 8$ derived in Ref.23 (we choose a broadening $\hbar \Gamma$ half that used in Ref.23). All the quantities $\left(\left\langle E_{K i n}\right\rangle, E_{p}, E_{g}\right.$ and $\left.e V_{\text {bias }}\right)$ are in unit $\hbar \Gamma$.

ferent interaction strengths $E_{p}$ as function of the bias voltage. First of all, we note that, regardless the values of $E_{p}$, for $V_{\text {bias }}=0$ all kinetic energy curves show $\left\langle E_{k i n}\right\rangle=0$. At equilibrium, we can say that the oscillator 'thermalizes' to the temperature of the electronic bath $\left(T_{e l}=0\right)$. We have also plotted two constant energy lines that specify the range of validity of our approximation, $E=\hbar \omega_{0} / 2 \sim k_{B} T_{D} / 2$ and $E=\hbar \Gamma$. At intermediate bias values, the curves show a departure from the common linear behaviour observed in the small bias regime, more evident as the interaction strength increases. The kinetic energy curves corresponding to $E_{p}=2.0$ and $E_{p}=3.0$ show an interesting plateau at intermediate bias where increasing the bias does not produce an increase of the average kinetic energy. Actually, at $E_{p}=3.0$, we find even a very slight decrease. We also note, in the same regime, that the velocity distribution probabilities are not gaussian.

\section{Limits of the adiabatic approach}

As mentioned, we can use the average kinetic energy of the oscillator to fix the range of validity of the

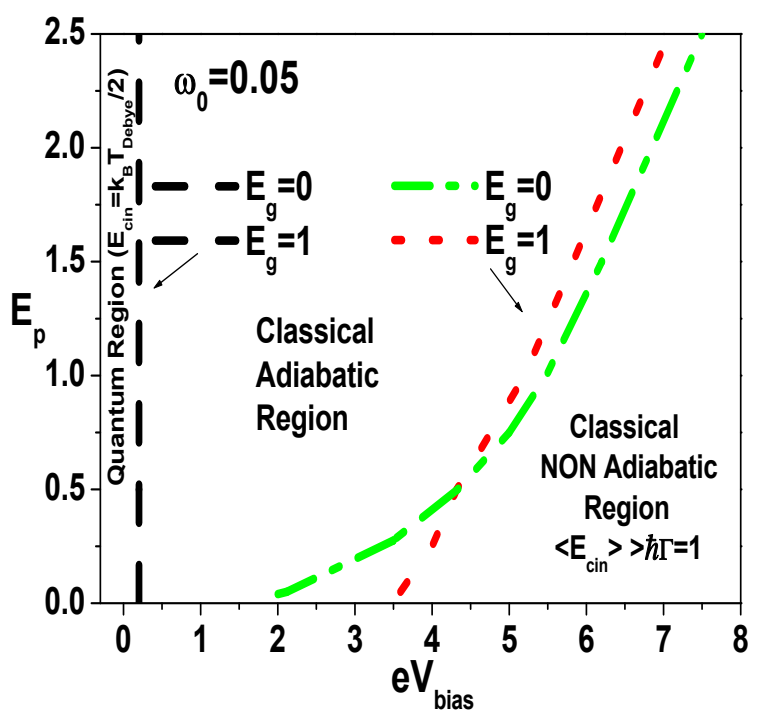

FIG. 5. (Color online). Diagram for the range of validity of classical approximation at fixed adiabatic ratio $\omega_{0} / \Gamma=0.05$ (the value of $\omega_{0}$ shown in the figure is in $\Gamma$ units). The dashed (black) line indicates the QR-CAR crossover for $E_{g}=0$ and $E_{g}=1$. The dotted (red) and dashed dotted (green) lines indicate the CAR-CNAR crossover for $E_{g}=0$ and $E_{g}=1$, respectively. $E_{p}, E_{g}$ and $e V_{\text {bias }}$ are expressed in unit $\hbar \Gamma$.

adiabatic approximation. If this energy is lower than the characteristic Debye temperature of the oscillator $\left\langle E_{K i n}\right\rangle<\hbar \omega_{0} / 2 \sim k_{B} T_{D} / 2$, we actually explore a region, as discussed in Ref.40, where quantum correlation effects can not be disregarded. We call this region Non Classical or Quantum Region (QR). If $k_{B} T_{D} / 2<\left\langle E_{K i n}\right\rangle<\hbar \Gamma$, that is the kinetic energy is lesser than the characteristic energy scale of the electronic degrees of freedom and simultaneously greater than characteristic Debye temperature, a huge number of vibrational quanta (phonons) are excited in the system. We call this region Classical Adiabatic (CAR). When the average kinetic energy of the oscillator exceeds the characteristic energy scale of the electron dynamics $\left\langle E_{\text {Kin }}\right\rangle>\hbar \Gamma$, we clearly are going beyond the limit of adiabatic approximation we start with. We define this region Classical Non Adiabatic (CNAR). We expect that in the CAR our approximation is very accurate. By using this data, we are now able to build up a diagram for the validity of classical approximation in the plane $\left(E_{p}-V_{\text {bias }}\right)$ for different values of gate voltages (Fig (5) and different adiabatic ratios (Fig [6). It is interesting to note that, in Fig 5 , the QR-CAR crossover line is almost independent from the gate voltage in the limit of small adiabatic ratio. Instead, the CAR-CNAR crossover line is slightly dependent from the gate voltage showing an enlargement of the CAR with $E_{g}$. Globally we note that, apart for the QR (small bias), the CAR enlarges as one increases the electron oscillator coupling.

As expected, as we increase the adiabatic ratio, the 


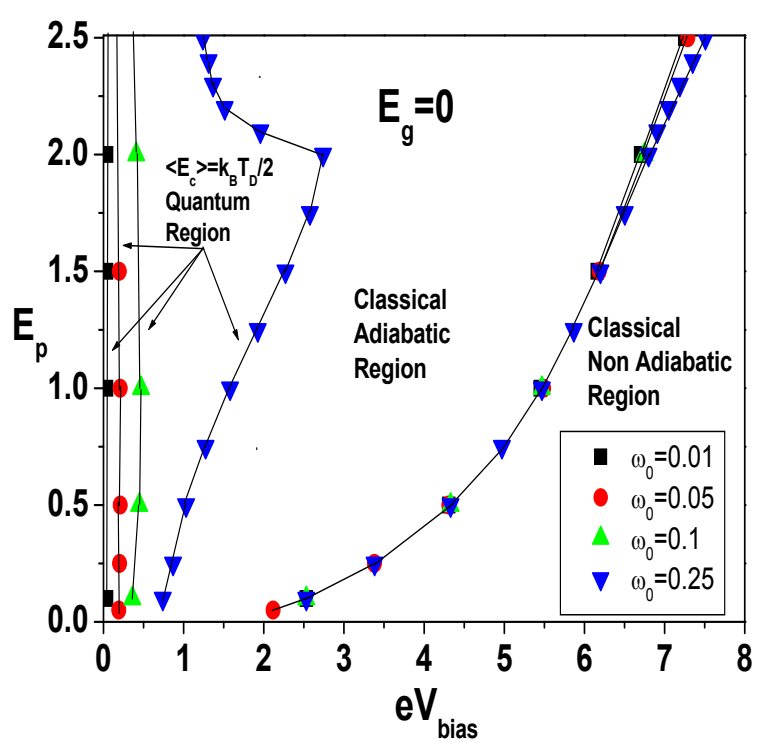

FIG. 6. (Color online). Diagram for the validity of classical approximation at fixed gate voltage $E_{g}=0$ (asymmetric static potential) for different adiabatic ratios $\omega_{0} / \Gamma=$ $0.01-0.05-0.1-0.25$ (the values of $\omega_{0}$ shown in the figure are in $\Gamma$ units). $E_{p}, E_{g}$ and $e V_{\text {bias }}$ are in $\hbar \Gamma$ units.

QR expands reaching great values of bias voltage $V_{\text {bias }}$, Fig6. In particular, observing the down-triangle (blue) curve (adiabatic ratio $\omega_{0} / \Gamma=0.25$ ), one notes that the QR-CAR crossover line reaches a "maximum" in correspondence of $E_{p} \simeq 2$ and $V_{\text {bias }} \simeq 2.8$. For $E_{p}<2$, the bias values identifying the QR-CAR crossover increase as the voltage increases. For couplings $E_{p}>2$, we note an inversion of this behaviour: the CAR starts to extend for a very large area of the diagram except for a narrow region at small bias (QR) and for a region at bigger bias values (CNAR). This means that, even for intermediate adiabatic ratios, we need sufficiently strong couplings $E_{p}$ in order to obtain a predominant CAR in the validity diagram. Moreover, this is due to the fact that the node between kinetic energy curves and the Debye line occurs in the non monotonic intermediate bias region (see Fig 4). On the other hand, the CAR-CNAR crossover line is almost independent from the adiabatic ratio (for not too large interaction strength). This is what we expect from physical grounds and constitutes a self-consistent check of our approximation.

\section{Electronic transport properties}

We can now analyze the electronic transport properties resulting from the average over the dynamical fluctuations of the oscillator motion. We first study the conductance-voltage curves as function of the EOC strength (Fig.7), then we show how the dynamical fluctuations strongly renormalize the infinite mass approxi-

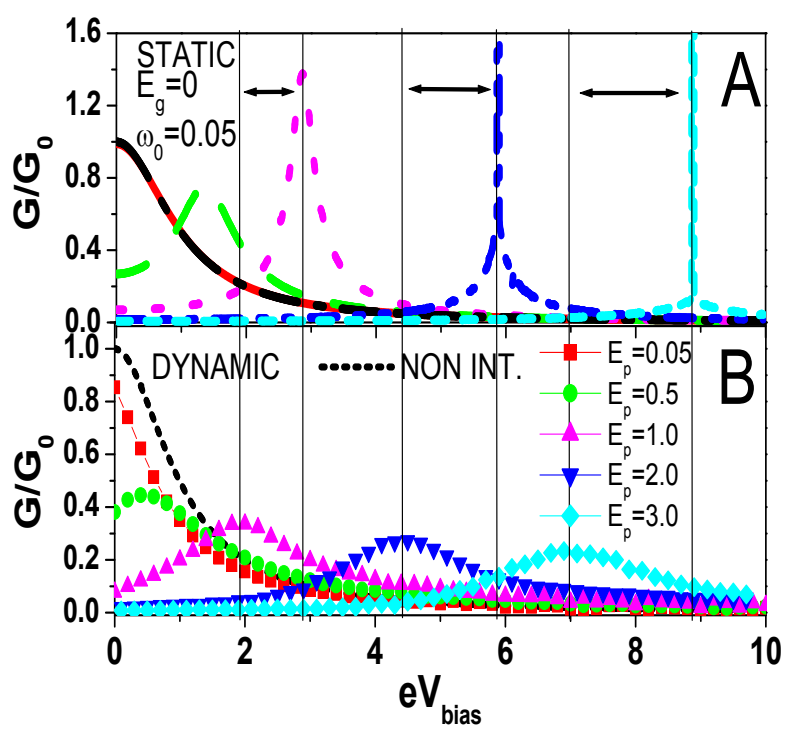

FIG. 7. (Color online). Panel A: Conductance (in units $G_{0}=$ $\left.\frac{e^{2}}{2 h}\right)$ in the static approximation as function of bias voltages, for $\omega_{0} / \Gamma=0.05, E_{g}=0$ and different interaction strengths $E_{p}=0.05,0.5,1.0,2.0,3.0$. Panel B: Dynamical correction to the conductance for the same parameter values of panel A. The value of $\omega_{0}$ shown in the figure is in $\Gamma$ units while all other quantities $\left(E_{g}, E_{p}\right.$ and $\left.e V_{\text {bias }}\right)$ are expressed in $\hbar \Gamma$ units.

mation results studying the I-V curves for different adiabatic ratios (Fig 8). Finally, we investigate the dependence of the kinetic energy and of the I-V characteristic as function of gate voltage studying the properties of the junction as function of an electric field orthogonal to the source-drain direction (Fig 9).

In Fig: 7 we show several conductance curves for different interaction strengths, $E_{p}=0.05-3$, at $\omega_{0} / \Gamma=0.05$ and $E_{g}=0$. The comparison between static (panel A) and dynamical (panel B) approximation is very interesting. The static solution shifts the non interacting resonance by a quantity proportional to the polaronic energy $E_{p}$ (panel A). As one can see, this effect strongly reduces the small bias conductance. The dynamical correction, on the other hand, reduces the polaronic shift compared to the static curves and also broadens (as a result of the very broad nonequilibrium distribution probabilities $P(x))$ the electronic resonance. In the intermediate bias regime, we note a strong enhancement of the conduction far from the electronic resonance where a very small current is observed in the static approximation. Moreover, including the dynamical fluctuations, the reduction of the small bias conductance is less pronounced. We note also that our dynamical approximation is close to the static solution in the small bias regime, while is substantially different in intermediate one. The dynamical corrections strongly renormalize the static results even for small adiabatic ratios. 


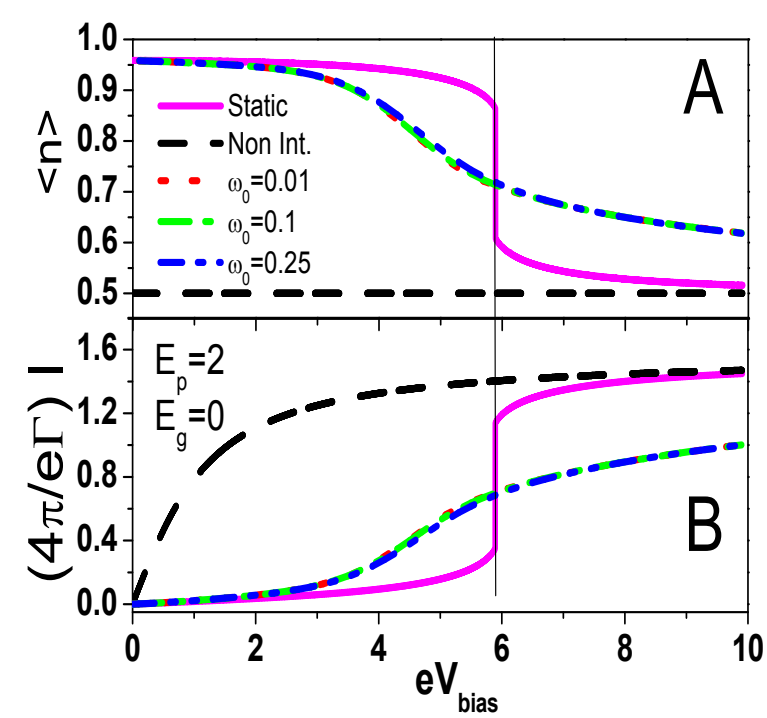

FIG. 8. (Color online). Panel A: Electronic occupation as function of bias voltages, for $E_{g}=0, E_{p}=2$ and different adiabatic ratios $\omega_{0} / \Gamma=0.05,0.25,0.5,1.0$. Panel B: Current voltage characteristic for the same value of the parameters of panel A. The values of $\omega_{0}$ shown in the figure are in $\Gamma$ units while all other quantities $\left(E_{g}, E_{p}\right.$ and $\left.e V_{\text {bias }}\right)$ are expressed in $\hbar \Gamma$ units.

We analyze in Fig 8 the behaviour of the electronic occupation (panel A) and current voltage characteristic (panel B) at strong coupling $E_{p}=2$, for different adiabatic ratios $\omega_{0} / \Gamma=0.01,0.1,0.25$ and at $E_{g}=0$. In the small bias regime, as a result of strong electron-oscillator interaction, the molecular level renormalizes itself far below the chemical potential of the leads. We note a large difference between the non interacting occupation value $(\langle\hat{n}\rangle \simeq 0.5)$ and the interacting one $(\langle\hat{n}\rangle \simeq 1)$. As one increases the bias voltage, many charges are pumped out the molecular 'dot'. In the large bias regime the stationary charge quantity in the molecular 'dot' reduces approaching the non interacting value $(\langle\hat{n}\rangle \simeq 0.5)$. The nonequilibrium broadening of the distribution probabilities $P(x)$, then, induces a strong reduction of the conduction threshold with respect to the static solution (solid magenta curve in Fig 8 panel B). We note a small variation of physical properties with respect to the adiabatic ratio at intermediate voltages, in the CAR in correspondence to non-gaussian regime of the distribution probabilities.

In many molecular transport experiments, one records the current or the conductance varying an electric field applied on the molecule (orthogonal to the source-drain direction) at fixed source-drain voltage. In the panel A of Fig $\underline{9}$, it is shown the current for different bias voltages at moderately small electron-oscillator coupling $\left(E_{p}=0.25\right)$ as function of gate voltage. In this regime we have no bistability in the model $\left(E_{p}<\hbar \Gamma\right)$. We note

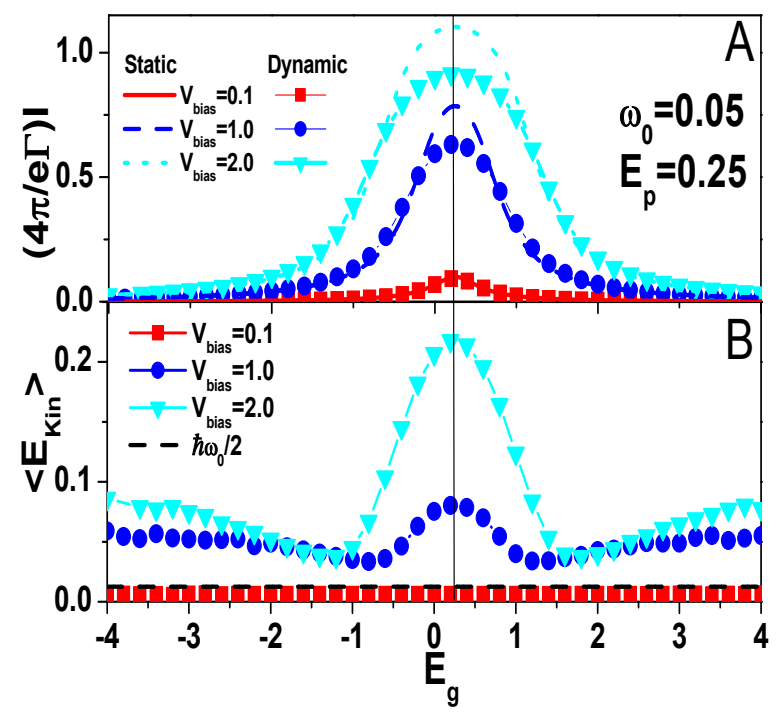

FIG. 9. (Color online). Panel A: Current as function of gate voltages, for $\omega_{0} / \Gamma=0.05, E_{p}=0.25$ and different bias voltages $V_{\text {bias }}=0.1,1.0,2.0$. Panel B: Plot of average kinetic energy as function of the gate for $V_{\text {bias }}=0.1,1.0,2.0$. The value of $\omega_{0}$ shown in the figure is in $\Gamma$ units, while $E_{g}$ and $E_{p}$ are expressed in $\hbar \Gamma$ units. $V_{\text {bias }}$ is expressed in $\hbar \Gamma / e$ units.

that the static and dynamical approximations agree in the small bias regime (solid and square lines in panel A of Fig (9). Increasing the bias voltage, the dynamical correction becomes more important showing a suppression of the current for small $E_{g}$. This effect is caused by the spectral weight broadening due to the average over the position distribution probabilities $P(x)$. From panel B of Fig 9 (square (red) line), we learn that, in the small bias regime, the kinetic energy is independent of the gate voltage, while, as the voltage increases, it shows a symmetric drop with respect to polaronic energy $E_{g}=E_{p}$, corresponding to the symmetric regime. The I-V curve also shares this symmetry. This effect can be explained observing that, when the 'bare' molecular level and the renormalized one are both in the bias window, the energy associated to the electronic current flow is more efficiently exchanged with the oscillator. When the electronic resonance is far above or below the chemical potential of the leads there is a less effective coupling between the oscillator and the electronic subsystem.

\section{THE TWO-SITE SSH MODEL}

The first step towards a more realistic description of a molecular junction is to consider a model Hamiltonian composed by two sites connected by an internal hopping $t$. In particular dimer molecules $\frac{38}{2}$ this hopping can be controlled by a vibrational mode which assists the electron tunneling through the two molecular sites. In this 
case, a guess for the molecular Hamiltonian is given by

$$
\hat{H}_{M o l}^{S S H}=E_{g}\left(\hat{d}_{1}^{\dagger} \hat{d}_{1}+\hat{d}_{2}^{\dagger} \hat{d}_{2}\right)-t(x)\left(\hat{d}_{1}^{\dagger} \hat{d}_{2}+\text { h.c. }\right),
$$

where we consider, as in the SSH model, an electron hopping

$$
t(x)=t-\alpha x
$$

depending linearly on the lattice displacement $x$ associated with the intermolecular vibrational mode. The molecular sites have a common energy $E_{g}$ and are described in terms of creation (annihilation) operators $\hat{d}_{i}^{\dagger}\left(\hat{d}_{i}\right), i=1,2$. The SSH model was indroduced to describe the transport properties of conducting polymers (e.g. polyacetylene ${ }^{29}$ ) and the two site case represents the shortest version of a molecular wire .30 A generalization of this two site model was proposed in Ref.32 for the study the electron transport of dimer molecules interacting with a single internal vibrational mode.

Most molecular devices studied experimentally so far ${ }^{2.3 .38}$ have been weakly coupled to the leads. This corresponds to the bare tunnel broadening $\hbar \Gamma$ of molecular electronic levels smaller that the energy required to excite one oscillator quantum (phonon) $\hbar \omega_{0}$. In the strongcoupling regime, when the electron-oscillator interaction energy $E_{p}$ exceeds $\hbar \omega_{0}$, the physics is governed by the Franck-Condon effect 19 -21 tron onto the molecule with the simultaneous emission or absorption of several phonons is more probable than elastic tunneling. The current as the function of voltage exhibits steps separated by $\hbar \omega_{0} / e \stackrel{22}{\longleftarrow}$ and the conductance shows phonon sidebands $s^{32}$

As in the AH model, we study here the case of slow phonons, $\omega_{0}<<\Gamma$, coupled to a molecular junction driven by a finite bias, in particular for $e V_{\text {bias }}>\hbar \omega_{0}$. As further approximation, we consider the dynamics of the vibrational mode "classical"'.

The structure of the SSH model is very interesting. The direct coupling of the electron-oscillator interaction to the intermolecular hopping $t$ suggests that the role of the dynamical fluctuations becomes crucial to determine the physical scenario. The total Hamiltonian is

$$
\hat{\mathcal{H}}_{T O T}=\hat{\mathcal{H}}_{e l-S S H}+H_{o s c},
$$

where

$$
\hat{\mathcal{H}}_{\text {el-SSH }}=\hat{H}_{M o l}^{S S H}+\hat{H}_{\text {Tun }}+\hat{H}_{\text {leads }},
$$

with $\hat{H}_{\text {leads }}$ and $\hat{H}_{\text {osc }}$ given by Eq.(3) and Eq.(4), respectively. The tunneling Hamiltonian $\hat{H}_{T u n}$ is given by

$\hat{H}_{\text {Tun }}=\sum_{k, L}\left(V_{k, L} \hat{c}_{k, L}^{\dagger} \hat{d}_{1}+\right.$ h.c. $)+\sum_{k, R}\left(V_{k, R} \hat{c}_{k, R}^{\dagger} \hat{d}_{2}+\right.$ h.c. $)$,

indicating that the left (right) lead is coupled only to the molecular site 1(2). In real space, the molecular Hamiltonian $\hat{H}_{M o l}^{S S H}$ is not diagonal. We therefore perform a

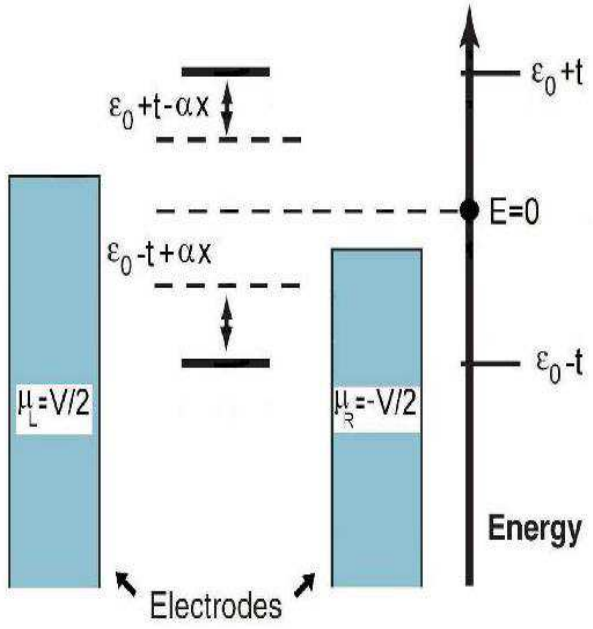

FIG. 10. (Color online). Sketch of junction within the SSH model in an energy scale.

transformation which diagonalizes the molecular isolated problem

$$
\begin{aligned}
& \hat{c}_{\gamma_{1}}^{\dagger}=\frac{\hat{d}_{1}^{\dagger}+\hat{d}_{2}^{\dagger}}{\sqrt{2}}, \\
& \hat{c}_{\gamma_{2}}^{\dagger}=\frac{\hat{d}_{1}^{\dagger}-\hat{d}_{2}^{\dagger}}{\sqrt{2}},
\end{aligned}
$$

with the same transformation for corresponding annihilation operators. This transformation leaves invariant $\hat{H}_{l e a d s}$ but changes $\hat{H}_{M o l}^{S S H}$ and $\hat{H}_{T u n}$. Explicitly we have

$$
\begin{aligned}
\hat{H}_{M o l}^{S S H} & =\varepsilon_{\gamma_{1}}(x) \hat{c}_{\gamma_{1}}^{\dagger} \hat{c}_{\gamma_{1}}+\varepsilon_{\gamma_{2}}(x) \hat{c}_{\gamma_{2}}^{\dagger} \hat{c}_{\gamma_{2}}, \\
\hat{H}_{\text {Tun }} & =\sum_{i=1,2}\left[\sum_{k}\left(\frac{V_{k, L}}{\sqrt{2}} \hat{c}_{k, L}^{\dagger} \hat{c}_{\gamma_{i}}+\text { h.c. }\right)\right. \\
& \left.+(-1)^{i-1} \sum_{k}\left(\frac{V_{k, R}}{\sqrt{2}} \hat{c}_{k, R}^{\dagger} \hat{c}_{\gamma_{i}}+\text { h.c. }\right)\right],
\end{aligned}
$$

where

$$
\begin{aligned}
& \varepsilon_{\gamma_{1}}(x)=\varepsilon+(t-\alpha x), \\
& \varepsilon_{\gamma_{2}}(x)=\varepsilon-(t-\alpha x) .
\end{aligned}
$$

As one can see, the above transformation allows us to take into account exactly the intermolecular hopping's effect. The molecular Hamiltonian $\hat{H}_{\text {Mol }}^{S S H}$ (Eq.(31)) is equivalent, at fixed $x$, to that of a non interacting two level system. In Fig 10 a schematic picture of the junction in an energy representation is shown. We observe that there are two electronic resonances, corresponding to a 'bonding' and 'anti-bonding' states whose position is renormalized by the electron-oscillator interaction.

From now on we work in the energy space for convenience. 
In the following, we will (subsection A) first analyze the coupled electron-oscillator problem within the $\mathrm{SSH}$ model in the limit of infinite mass for the oscillator. Then, we will construct, as done in AH model, the stochastic Langevin equation for the dynamics of the oscillator. In the subsection B we will describe the numerical results.

\section{A. Out of equilibrium Born-Oppenheimer approximation: infinite mass (static) case}

As in AH model, performing the limit $m \mapsto \infty$, at zero-order static approximation, we neglect the kinetic energy of the oscillator. The electronic dynamics, with the oscillator displacement $x$ as a free parameter, is therefore equivalent in the energy space to a non-interacting two level problem with energy levels renormalized by the 'polaronic' shift $-\alpha x$, Eq.(33). In what follows, we consider the case of symmetric coupling of the molecule to the leads $\hbar \Gamma_{L}=\hbar \Gamma_{R}$ in the wide-band approximation. Here, we briefly show how to calculate the generalized potential of the oscillator coupled to the double 'dot' molecular junction.

Within the Keldysh formalism, we use the equation of motion approach to calculate the molecular Green functions in stationary nonequilibrium conditions. In the zero order static approximation, we have the following equation of motion for the molecular retarded Green function

$$
\begin{aligned}
& \left(\begin{array}{cc}
\imath \hbar \frac{\partial}{\partial t}-\varepsilon_{\gamma_{1}}(x)+\imath \frac{\hbar \Gamma_{L}+\hbar \Gamma_{R}}{4} & \imath \frac{\hbar \Gamma_{L}-\hbar \Gamma_{R}}{4} \\
\imath \frac{\hbar \Gamma_{L}-\hbar \Gamma_{R}}{4} & \imath \hbar \frac{\partial}{\partial t}-\varepsilon_{\gamma_{2}}(x)+\imath \frac{\hbar \Gamma_{L}+\hbar \Gamma_{R}}{4}
\end{array}\right) \\
& \times\left(\begin{array}{cc}
G_{1,1}^{r}\left(t, t^{\prime}\right) & G_{1,2}^{r}\left(t, t^{\prime}\right) \\
G_{2,1}^{r}\left(t, t^{\prime}\right) & G_{2,2}^{r}\left(t, t^{\prime}\right)
\end{array}\right)=\delta\left(t-t^{\prime}\right)\left(\begin{array}{ll}
1 & 0 \\
0 & 1
\end{array}\right),
\end{aligned}
$$

which acquires a $2 \times 2$ matrix structure. A similar equation is valid also for the advanced Green function. In the hypothesis of symmetric coupling with the leads, one obtains two separate problems for the molecular energy levels $\varepsilon_{\gamma_{1}}(x)$ and $\varepsilon_{\gamma_{2}}(x)$, respectively. The diagonal elements of the retarded Green function in Fourier space are

$$
G_{i, i}^{r}(\omega, x)=\frac{1}{\hbar \omega-\varepsilon_{\gamma_{i}}(x)+\imath\left(\frac{\hbar \Gamma^{L}+\hbar \Gamma^{R}}{4}\right)}, \quad i=1,2
$$

while the non diagonal terms are zero.

The lesser matrix Green function is instead given by

$$
\begin{gathered}
G^{<}(\omega, x)= \\
\imath \frac{\hbar \Gamma}{4}\left(\begin{array}{cc}
\left(n_{L}+n_{R}\right)\left|G_{1,1}^{r}\right|^{2} & \left(n_{L}-n_{R}\right) G_{1,1}^{r} G_{2,2}^{a} \\
\left(n_{L}-n_{R}\right) G_{2,2}^{r} G_{1,1}^{a} & \left(n_{L}+n_{R}\right)\left|G_{2,2}^{r}\right|^{2}
\end{array}\right)
\end{gathered}
$$

where, for sake of simplicity, we have dropped the frequency $\omega$ and the displacement $x$ dependence. The diagonal terms of the lesser Green function are directly related to the electron ' 'densities' ' (these obviously not correspond to the densities in real space)

$$
\left\langle\hat{n}_{\gamma_{i}}\right\rangle(x)=\frac{1}{2}+\frac{1}{2 \pi} \sum_{\alpha=R, L} \arctan \left[\frac{\mu_{\alpha}-\varepsilon_{\gamma_{i}}(x)}{\hbar \Gamma / 4}\right], \quad i=1,2 .
$$

For sake of clarity, we show here that the population in real space of the left and right molecular sites are expressed in terms of lesser Green functions (Eq.(36)

$$
\left\langle\hat{n}_{i}\right\rangle(x)=\frac{1}{2} \int \frac{d \omega}{2 \pi \imath}\left(G_{1,1}^{<}+G_{2,2}^{<}+(-1)^{i+1}\left(G_{1,2}^{<}+G_{2,1}^{<}\right)\right),
$$

where $i=1,2$ (site 1 is the left site, site 2 the right one).

The force exerted on the oscillator is given by

$$
F_{S S H}=-k x+\alpha\left(\left\langle\hat{n}_{\gamma_{1}}\right\rangle-\left\langle\hat{n}_{\gamma_{2}}\right\rangle\right)(x) .
$$

Taking care of Eq.(37) and Eq.(39), one can straightforwardly compute the expression of the generalized potential in nonequilibrium conditions $\left(\mu_{R}=-e V_{\text {bias }} / 2\right.$, $\mu_{L}=e V_{\text {bias }} / 2$ )

$$
\begin{aligned}
& V_{S S H}(x)=\frac{1}{2} k x^{2}-\sum_{\alpha=L, R} \sum_{i=1,2}\left[\frac{\mu_{\alpha}-\varepsilon_{\gamma_{i}}(x)}{2 \pi} \times\right. \\
& \arctan \left(\frac{\mu_{\alpha}-\varepsilon_{\gamma_{i}}(x)}{\hbar \Gamma / 4}\right)-\frac{\hbar \Gamma}{16 \pi} \ln \left[16\left(\mu_{\alpha}-\varepsilon_{\gamma_{i}}(x)\right)^{2}\right. \\
& \left.\left.+(\hbar \Gamma)^{2}\right]\right] .
\end{aligned}
$$

This generalized oscillator potential depends parametrically on the new electronic energy scale introduced in the problem: the intermolecular hopping $t$ ' 'hidden' ' in $\varepsilon_{\gamma_{i}}(x)$, see Eq. 333. Furthermore, it depends on the polaron energy, $E_{p}$, the gate voltage $E_{g}$, and the bias Vias.

In Fig 11 we present some features of the generalized potential $V_{S S H}(x)$ which will allow us to understand the effect of the nonequilibrium electronic system on the "static" stretching of the oscillator (solutions of the equation $F_{S S H}=0$ ). Moreover, this will help us to clarify the role of the dynamical effects in the transport properties that we will show later.

We focus here on the weak coupling $\left(E_{p} / \hbar \Gamma<<1\right)$ regime where moreover the intermolecular hopping $t$ is larger than the coupling $\hbar \Gamma$ of the molecule with the leads. In the panel $\mathrm{A}$ we show the generalized potential of the SSH model at fixed EOC strength, $E_{p}=0.2$, intermolecular hopping $t=2.0$, as function of the bias voltage $V_{\text {bias }}$. One can observe that, as the bias increases, the position of generalized potential minimum goes from $x \simeq-1$ (corresponding to $\left\langle n_{\gamma_{1}}\right\rangle-\left\langle n_{\gamma_{2}}\right\rangle \simeq-1$ ) to $x \simeq 0$ (corresponding to $\left\langle n_{\gamma_{1}}\right\rangle-\left\langle n_{\gamma_{2}}\right\rangle \simeq 0$ ). The oscillator switches from a full stretching configuration $(x \simeq-1)$ to a no-stretching one $(x \simeq 0)$. At equilibrium (solid (black) curve of Panel A of Fig[11), we have a physical situation where the renormalized anti-bonding electron 


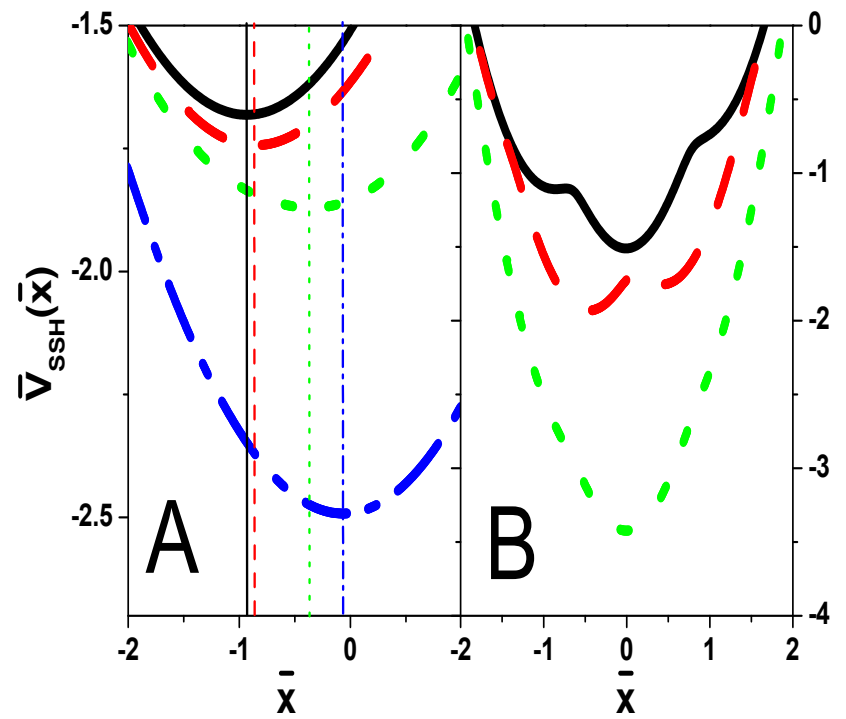

FIG. 11. (Color online). Panel A:Spatial dependence of the dimensionless generalized static potential $\bar{V}_{S S H}(\bar{x})$ at $\omega_{0} / \Gamma=$ $0.1, E_{p}=0.2$, gate voltage $E_{g}=0$, intermolecular hopping $t=2.0$, for different values of the bias voltage: $V_{\text {bias }}=0.0$ ( solid (black) curve), $V_{\text {bias }}=3.5$ (dashed (red) curve), $V_{\text {bias }}=$ 4.0 (dotted (green) curve), $V_{\text {bias }}=6.0$ (dashed dotted (blue) curve). The vertical lines indicate the position of the minima of the potential. Panel B: Same as above for $\omega_{0} / \Gamma=0.1, E_{p}=$ $1.4, t=0.2$, gate voltage $E_{g}=2$ and different values of the bias voltage: $V_{\text {bias }}=0$ (solid (black) curve), $V_{\text {bias }}=4$ (dashed (red) curve), $V_{\text {bias }}=8$ (dotted (green) curve). The potential is expressed in $\hbar \Gamma$ units $\left(\bar{V}_{S S H}=V_{S S H} / \hbar \Gamma\right)$. $V_{\text {bias }}$ values are expressed in $\hbar \Gamma / e$ units, where $e$ is the electron charge. $E_{g}, E_{p}$ and $t$ are expressed in $\hbar \Gamma$ units. The dimensionless position variable $\bar{x}$ is defined as $\bar{x}=x / x_{0}$ with $x_{0}=\frac{\lambda}{m \omega_{0}^{2}}$.

level $\varepsilon_{\gamma_{1}}(x)$ is above the chemical potential of both leads, while the bonding one $\varepsilon_{\gamma_{2}}(x)$ is below them. The classical "spring"' is fully compressed $(x \simeq-1)$ and this corresponds in real space to molecular sites half-filled $\left(\left\langle n_{1}\right\rangle \simeq\left\langle n_{2}\right\rangle \simeq 0.5\right)$. Studying the electronic populations of left (1) and right (2) molecular sites (Eq.(38)), one can observe that, if we increase the bias voltage, the left site starts to empty, while the right one populates, reaching, for $e V_{\text {bias }}^{*} / 2 \simeq t-\alpha x\left(V_{\text {bias }}^{*}\right.$ ) (hopping value properly renormalized), a small difference of population roughly equal to $\left\langle n_{1}\right\rangle-\left\langle n_{2}\right\rangle \simeq-0.1$. For sufficiently large bias, the molecular level populations tend again to the common value 0.5 . As we shall see in next section, the inclusion of the dynamical effects allows to clarify the physical picture arising from the above description, in terms of an energy balance between the electronic and oscillator subsystems.

At static level, it is also interesting to discuss the extremely strong coupling regime $E_{p}>\hbar \Gamma>t$, for gate voltage $E_{g}=2.0$ (panel B). In this case, at equilibrium, we are describing a physical situation where the renormalized bonding and anti-bonding electron levels are both above the chemical potential $\left(V_{\text {bias }}=0\right)$. The molecular sites in real space are both almost empty $\left(\left\langle n_{1}\right\rangle \simeq\left\langle n_{2}\right\rangle \simeq 0\right)$, and the oscillator is in a no-stretching configuration $x \simeq 0$ (solid (black) curve). Increasing the bias voltage, the generalized potential develops different minima. At intermediate bias, one can observe two asymmetric minima near $x \simeq-0.5$ and $x \simeq 0.5$, separated by a potential barrier. In this regime, the minimum corresponding to $x \simeq-0.5$ prevails (dashed (red) curve) and the non-interacting real space populations $\left\langle n_{1}\right\rangle$ and $\left\langle n_{2}\right\rangle$ are asymmetrically distributed between the two sites $\left(\left\langle n_{1}\right\rangle \simeq 0.8,\left\langle n_{2}\right\rangle \simeq 0.2\right)$. Instead, the interacting real space populations have the same value $\left\langle n_{1}\right\rangle \simeq\left\langle n_{2}\right\rangle \simeq 0.25$, corresponding to a very large current-carrying configuration. In the large bias regime only the minimum $x \simeq 0$ corresponding to a small-current-carrying configuration survives. Including the interaction effects, the left site results almost filled $\left\langle n_{1}\right\rangle \simeq 0.9$, while the right one almost empty $\left\langle n_{2}\right\rangle \simeq 0.1$, showing that, as result of the strong electron-phonon interaction, the bias voltage does not manage to deplete both molecular sites. As we shall see later, the features of the static potential obtained in this case determine the possibility to observe in the I-V a strong Negative Differential Resistance, when the dynamical effects of the oscillator are neglected.

\section{B. Adiabatic Approximation: calculation of damping and fluctuating term}

As we have discussed after the Eq.(34), the assumption of symmetric coupling to the leads allows to disentangle in the energy space the problem for the molecular bonding and anti-bonding levels $\varepsilon_{\gamma_{i}}(x)$. Repeating site-by-site the construction introduced in the previous section for AH model, we can straightforwardly set for our two site SSH model a Langevin equation for the oscillator dynamics, very similar to that derived in $\mathrm{AH}$ model. The new coefficients, $F(x), A(x)$ and $D(x)$ are given by

$$
\begin{aligned}
F(x) & =-k x+\lambda \frac{1}{2 \pi} \sum_{\alpha=R, L} \sum_{i=1,2} \arctan \left[\frac{\mu_{\alpha}-\varepsilon_{\gamma_{i}}(x)}{\hbar \Gamma / 4}\right], \quad(41) \\
A(x) & =\frac{16 \hbar k E_{p}}{\pi \hbar^{2} \Gamma^{2}} \sum_{\alpha=L,-R} \sum_{i=1,2}\left(\frac{1}{\left[\left(\frac{\mu_{\alpha}-\varepsilon_{\gamma_{i}}(x)}{\hbar \Gamma / 4}\right)^{2}+1\right]^{2}}\right), \quad(42) \\
D(x) & =\frac{k E_{p}}{\pi} \int d \omega\left[G_{1,1}^{<} G_{1,1}^{>}+G_{2,2}^{<} G_{2,2}^{>}+2 G_{1,2}^{<} G_{2,1}^{>}\right]= \\
& =\frac{2 k E_{p}}{\pi \Gamma} \sum_{\alpha=L,-R} \sum_{i=1,2}\left\{\left(\arctan \left(\frac{\mu_{\alpha}-\varepsilon_{\gamma_{i}}(x)}{\hbar \Gamma / 4}\right)\right.\right. \\
& \left.+\frac{\frac{\mu_{\alpha}-\varepsilon_{\gamma_{i}}(x)}{\hbar \Gamma / 4}}{\left[\left(\frac{\mu_{\alpha}-\varepsilon_{\gamma_{i}}(x)}{\hbar \Gamma / 4}\right)^{2}+1\right]}\right)+4\left(\frac{1}{\left(\frac{\varepsilon_{\gamma 1}(x)-\varepsilon_{\gamma_{2}}(x)}{\hbar \Gamma / 4}\right)^{2}+4}\right) \times
\end{aligned}
$$




$$
\begin{aligned}
& {\left[\arctan \left(\frac{\mu_{\alpha}-\varepsilon_{\gamma_{i}}(x)}{\hbar \Gamma / 4}\right)+\frac{(-1)^{i}}{\left(\frac{\varepsilon_{\gamma 1}(x)-\varepsilon_{\gamma_{2}}(x)}{\hbar \Gamma / 4}\right)} \times\right.} \\
& \left.\left.\ln \left(1+\left(\frac{\mu_{\alpha}-\varepsilon_{\gamma_{i}}(x)}{\hbar \Gamma / 4}\right)^{2}\right)\right]\right\},
\end{aligned}
$$

where in the first line of Eq.(43) we have dropped the frequency $\omega$ and the displacement $x$ dependence in the Green functions. We end this section briefly discussing some of the peculiarities of the damping function $A(x)$ and of the fluctuating term $D(x)$. As regards the damping term (panel A and C in Fig.(12)), one can observe that is located in suitable points and is strongly space dependent. It is interesting to note that, as in the $\mathrm{AH}$ model case, it survives also for $V_{\text {bias }}=0$ (solid (black) curves in Panels A-C). In this case, one can also note that, for $E_{p}<<t$ (panel A), $A(x)$ is almost zero in the interval mostly explored in the dynamics $(-2<x<2)$, while, for $E_{p}>t$ (panel C), shows two pronounced peaks in that interval. As one can see, the position of $A(x)$ 's maxima is strongly bias dependent.

As concerns the fluctuating term (Panels B and D in Fig.(12)), one can note that, as in the AH model, it is identically zero at equilibrium $\left(V_{\text {bias }}=0\right)$. When the bias increases, it becomes almost different from zero in the region mostly explored in the dynamics $(-2<x<2)$. In Panel B of Fig 12 one can observe that the spatial extension of the fluctuating term increases as the bias increases, while, in Panel D, in the interesting strong coupling regime $\left(E_{p}>>t\right)$, it shows a maximum for $x=0$, the no-stretching equilibrium state of the oscillator. It is important to stress again here that the space dependence of these terms determines the non-gaussian character of the distribution probabilities $P(x)$ and $P(v)$ of the oscillator.

\section{Analysis of Numerical results}

As done for the AH model, we here show the results arising from the numerical simulation of the Langevin equation of the SSH model. We evaluate the fundamental ingredients of the adiabatic approximation: the distribution probabilities for the oscillator. These allow us to calculate the dynamical properties of the oscillator (average kinetic and potential energy) as well as the electronic transport properties of the molecular junction.

\section{Study of the average kinetic energy of the oscillator and limits of the Adiabatic approach}

First of all, we study the behaviour of velocity distribution probabilities $P(v)$ resulting from the solution of the Langevin equation associated to the SSH model. As in AH model, we have verified that in the small bias regime, regardless the value of the gate voltages $E_{g}$, the

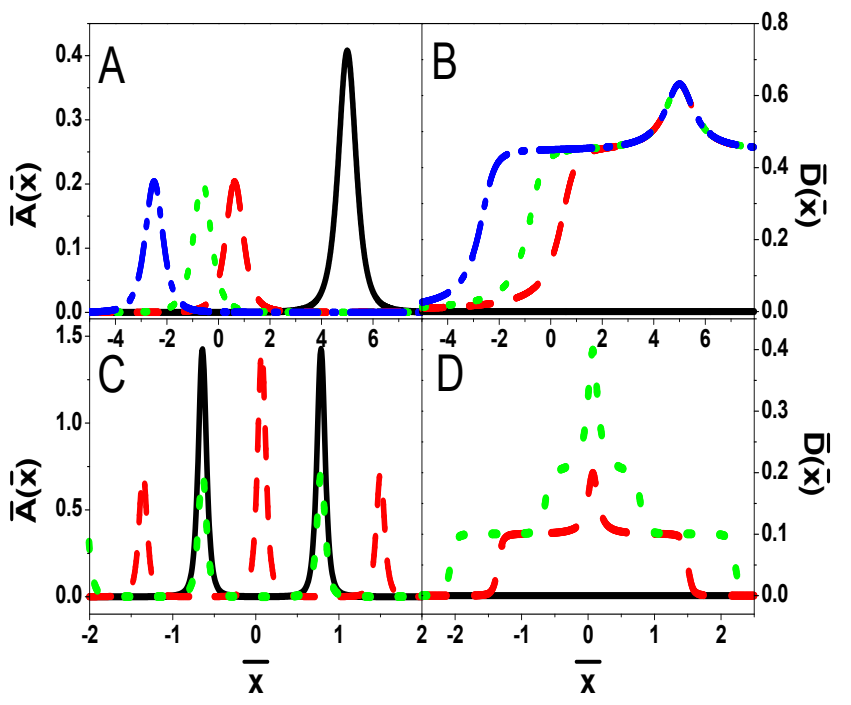

FIG. 12. (Color online). Panels A-B: Spatial dependence of the dimensionless friction coefficient $\bar{A}(\bar{x})$ and fluctuating term $\bar{D}(\bar{x})$ at $\omega_{0} / \Gamma=0.1, E_{p}=0.2$, gate voltage $E_{g}=0$, intermolecular hopping $t=2.0$, for different values of the bias voltage: $V_{\text {bias }}=0.0$ (solid (black) curve), $V_{\text {bias }}=3.5$ (dashed (red) curve), $V_{\text {bias }}=4.0$ (dotted (green) curve), $V_{\text {bias }}=6.0$ (dashed dotted (blue) curve). Panels C-D: Same as above for $\omega_{0} / \Gamma=0.1, E_{p}=1.4, t=0.2$, gate voltage $E_{g}=2$ and different values of the bias voltage: $V_{\text {bias }}=0$ (solid (black) curve), $V_{\text {bias }}=4$ (dashed (red) curve), $V_{\text {bias }}=8$ (dotted (green) curve). The friction coefficient is expressed in $m \omega_{0}$ units $\left(\bar{A}=A / m \omega_{0}\right)$ while the fluctuating term in $\lambda^{2} / \omega_{0}$ units, $\left(\bar{D}=D /\left(\lambda^{2} / \omega_{0}\right)\right) . V_{\text {bias }}$ values are expressed in $\hbar \Gamma / e$ units, where $e$ is the electron charge. $E_{g}, E_{p}$ and $t$ are expressed in $\hbar \Gamma$ units. The dimensionless position variable $\bar{x}$ is defined as $\bar{x}=x / x_{0}$ with $x_{0}=\frac{\lambda}{m \omega_{0}^{2}}$.

electron-oscillator coupling $E_{p}$ and the hopping $t$, the $d i$ mensionless velocity distribution probabilities $P(v)$ are gaussian. The introduction of a new energy scale in the problem does not much modify the physical picture we obtained in AH model in the small bias regime: the nonequilibrium electronic bath behaves like a conventional bath for the oscillator with an effective temperature linearly proportional to the bias voltage. In particular, in the SSH model case, it is worth noticing that the average kinetic energy exhibits a slope twice that found in the AH model. This is a consequence of the transformation Eq. (30) we have applied on the total Hamiltionian, that renormalized the tunneling amplitudes with the leads, $V_{k, \alpha} \mapsto V_{k, \alpha} / \sqrt{2}$. From the physical point of view, we find that the two electronic channels independently contribute to the oscillator effective temperature, showing that the problem is equivalent to the sum of two singlesite junctions.

As we increase the bias voltage, the $\left(\log (P(v))\right.$ vs. $\left.v^{2}\right)$ plot starts to deviate from a linear trend, so that, even in SSH case, the oscillator dynamics cannot be simply 


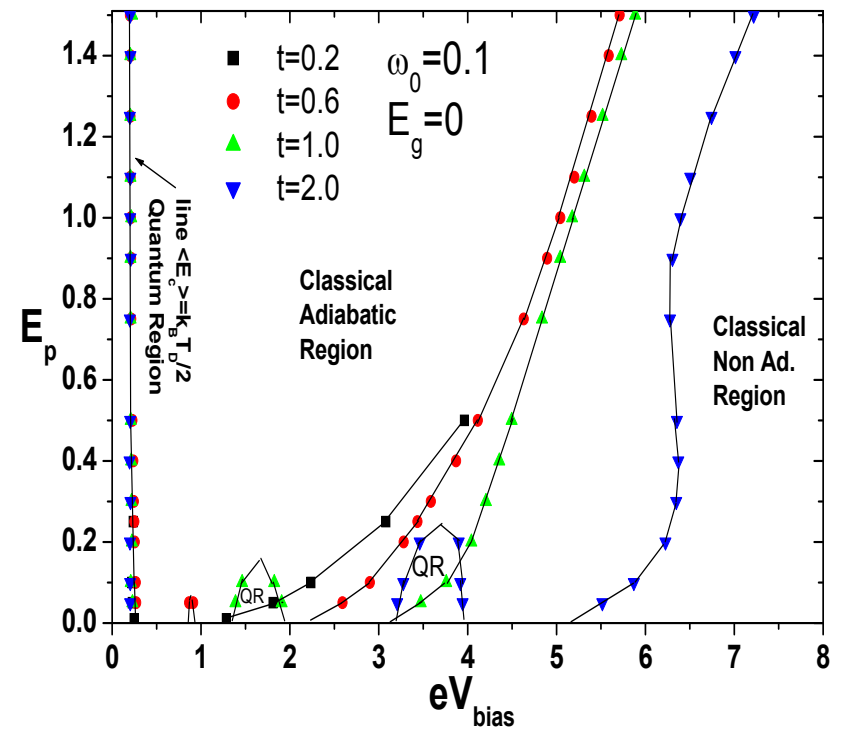

FIG. 13. (Color online). Diagram for the validity of classical approximation at fixed adiabatic ratio $\omega_{0} / \Gamma=0.1, E_{g}=0$, for different values of intermolecular hopping $t=0.2-0.6-$ $1.0-2.0$. The value of $\omega_{0}$ shown in the figure is in $\Gamma$ units, while all other quantities $\left(E_{g}, E_{p}, t\right.$ and $\left.e V_{\text {bias }}\right)$ are expressed in $\hbar \Gamma$ units.

reduced to an effective temperature in the intermediate bias regime.

We also find that, for $0<t / E_{p}<1$ and up to very large values of the bias voltage, the average kinetic energy shows a behaviour qualitatively similar to that of AH model (Fig, 4n). In this regime, we can conclude that the dynamical fluctuations of the oscillator motion do not 'see' the double 'dot' structure of the electronic molecular junction. If $t / E_{p}>>1$, as we will discuss later, the average kinetic energy shows an interesting non monotonic behaviour in the intermediate bias regime (see below, Fig 14).

The systematic analysis of the average kinetic energy allows us to build up a diagram for the validity of classical approximation in the plane $\left(E_{p}-V_{\text {bias }}\right)$, as done for $\mathrm{AH}$ model (Fig 13). In particular, we study the validity diagram for different values of intermolecular hopping $t$ and at fixed adiabatic ratio and gate voltage. In this case, it is interesting to note that QR-CAR crossover line is almost independent by the intermolecular hopping in the limit of small adiabatic ratio. Joining together the results obtained for the $\mathrm{AH}$ validity diagrams (Fig 5 and Fig 6 ), we can conclude that, in the limit of very small adiabatic ratios, the QR-CAR crossover line is completely independent by the other energy scales considered in the problem. The CAR-CNAR crossover line is instead slightly dependent on $t$ showing the expansion of the CAR. As the intermolecular hopping $t$ increases, bigger values of bias voltage are needed to get average kinetic energy values greater than energy coupling to the leads, $\left\langle E_{K i n}\right\rangle>\hbar \Gamma$.

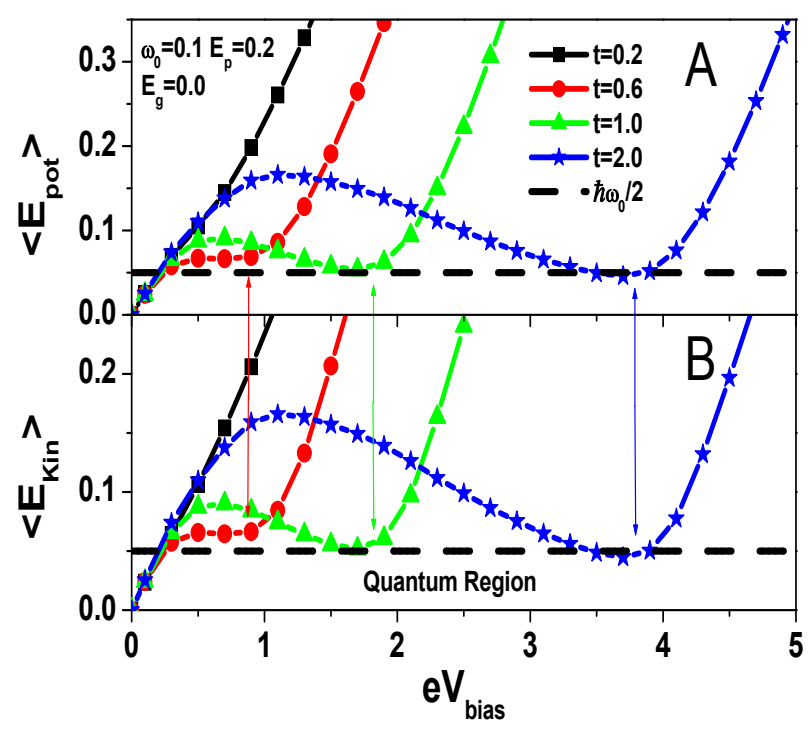

FIG. 14. (Color online). Panel A: Average potential energy as function of the bias for different values of intermolecular hopping $t=0.2-0.6-1.0-2.0$. Panel B: Average kinetic energy as function of the bias for values of intermolecular hopping as in panel A. We note that the introduction of new energy scale makes the overall energy $\langle E\rangle$ a decreasing function of the voltage for intermediate values. The value of $\omega_{0}$ shown in the figure is in $\Gamma$ units, while all other quantities $\left(E_{g}, E_{p}\right.$, $\left\langle E_{\text {Kin }}\right\rangle,\left\langle E_{\text {pot }}\right\rangle, k_{B} T, t$ and $\left.e V_{\text {bias }}\right)$ are expressed in $\hbar \Gamma$ units.

In this case, the intermolecular hopping $t$ plays the same role as the gate in AH model (see, Fig 5).

A new feature which was not observed in the AH model is the appearance of small QR for sufficiently small coupling $E_{p}$, at intermediate bias voltages (Fig 13). For strong enough electron-phonon coupling $E_{p}$, these regions disappear. This feature can be understood analyzing the behaviour the average kinetic energy $\left\langle E_{K i n}\right\rangle$ for the parameters characterizing the QR observed at intermediate bias. As it is clear form Fig 14, $\left\langle E_{K i n}\right\rangle$ can decrease significantly at intermediate $V_{\text {bias }}$. The effect becomes less and less evident decreasing the intermolecular hopping and disappears at $t=0.2$. It is interesting to note that the potential energy curves show almost the same trend (see Fig 14, panel A). Therefore, for sufficiently large $t$ and small $E_{p}$, the oscillator overall energy decreases as a function of bias voltage.

The behaviour of the average energy of the oscillator as function of bias voltage is determined by net balance of energy exchanged by the junction: after an increasing trend in the small bias regime, where the energy pumped by the bias exceeds that ceased to the electrons by the oscillator, the decreasing behaviour in the intermediate bias regime is due to the opposite physical mechanism: the energy ceased to the electron system by the oscillator exceeds that pumped by the bias.

This "transition" occurs for that particular range 
of bias voltages where the molecular energy levels are going through the bias window, with a resulting strong current enhancement (electronic resonance). In particular, when the electron molecular levels enter the bias window completely, in the case of symmetric bias unbalance and for $E_{g}=0$, we expect that the electronic conductance reaches its maximum. Remarkably, comparing Fig 15] and Fig 14, one can observe that the conductance maxima correspond to kinetic energy minima, shifted by a quantity close to the EOC strength $\alpha$. Physically, as a consequence of the $\mathrm{SSH}$ coupling with the oscillator, the current enhancement is followed by a strong effective absorption of energy of the electron system from the oscillator.

\section{Electronic transport properties}

In order to evaluate the current through the molecular system in SSH model, we use the Meir-Wingreen formula 39 for non interacting molecular levels, specialized to our two-level case

$$
I(x)=\frac{e}{\hbar} \int \frac{d \hbar \omega}{2 \pi}\left(f_{L}(\omega)-f_{R}(\omega)\right) \operatorname{Tr}\left\{\mathbf{G}^{a} \boldsymbol{\Gamma}_{L} \mathbf{G}^{r} \boldsymbol{\Gamma}_{R}\right\},
$$

where the matrices $\Gamma_{L / R}$ are given by

$$
\boldsymbol{\Gamma}_{L}=\frac{\hbar \Gamma}{4}\left(\begin{array}{ll}
1 & 1 \\
1 & 1
\end{array}\right), \quad \boldsymbol{\Gamma}_{R}=\frac{\hbar \Gamma}{4}\left(\begin{array}{cc}
1 & -1 \\
-1 & 1
\end{array}\right)
$$

and bold $\mathbf{G}^{r, a}$ indicate retarded (advanced) matrix green functions $(\mathrm{Eq} 35)$. We have explicitly indicated that the current depends on the deformation $x$ of the oscillator, so that it has to be averaged over the probability distribution function $P(x)$.

Here, we focus on two particular physical regimes, previously discussed in the analysis of the static approximation: the weak coupling $\left(E_{p}<<\hbar \Gamma\right)$ and the strong coupling $\left(\hbar \Gamma<<E_{p}\right)$ limits, varying arbitrarily the intermolecular energy scale $t$. As we shall see, in both regimes, the direct coupling of the electron-oscillator interaction to the intermolecular hopping makes the role of the dynamical fluctuations crucial to determine correct results.

In the weak coupling regime, it is interesting to observe that, as in the AH model, the dynamical corrections renormalize and broaden the electronic resonances (Fig 15) with respect to the static solution. In particular, in panel A of Fig 15, we note that the static approximation exceeds the maximum value of non interacting conductance and shows a region of negative conductance at intermediate bias. However, when the dynamical contributions are included (square (green) curve), the effect on the conductance is dramatic washing out all the structures observed in the static approximation. More interesting are the cases of panels B-C-D of Fig.15] where again the static approximation shows the spurious result

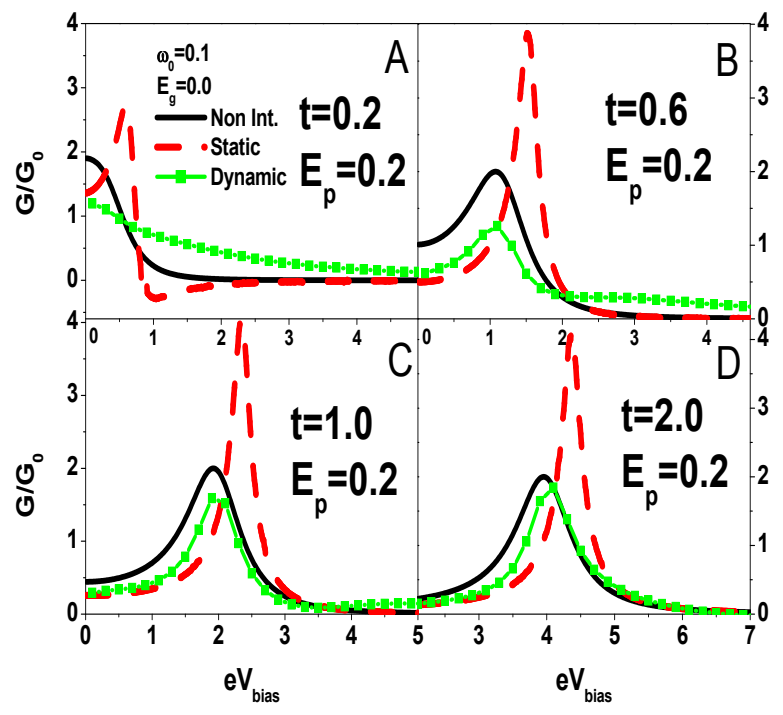

FIG. 15. (Color online). Panel A-D: Conductance as function of the bias at $\omega_{0} / \Gamma=0.1, E_{g}=0.0$, interaction strength $E_{p}=$ 0.2 , for different values of intermolecular hopping $t=0.2-$ $0.6-1.0-2.0$. The solid (black) line indicates non interacting curve. The dashed (red) line and the square (green) lines refer to the static and the dynamical approximation, respectively. The value of $\omega_{0}$ shown in the figure is in $\Gamma$ units, while all other quantities $\left(E_{g}, E_{p}, t\right.$ and $\left.e V_{\text {bias }}\right)$ are expressed in $\hbar \Gamma$ units.

of conductance greater than $2 G_{0}$, while the dynamical approximation renormalizes and broadens the peak of conductance to bias values where the static approximation shows small electric conduction. Even in the weak coupling regime, the inclusion of the dynamical fluctuations is essential to obtain correct results for the electronic conduction.

Finally, we examine the electronic transport properties in the strong coupling regime $\left(E_{p}>>\hbar \Gamma\right)$, where moreover $\hbar \Gamma>>t$. In this case, we expect strongly non linear behaviour of I-Vs in the infinite mass (static) limit for the oscillator. In Fig 16 we show the current voltage characteristic for strong interaction, $E_{p}=1.4$, at fixed adiabatic ratio $\omega_{0} / \Gamma=0.1$, gate voltage $E_{g}=2.0$ and for different small values of intermolecular hopping $t<<\hbar \Gamma$ $(t=0.15$ (black) dashed, $t=0.2$ (red) dashed dotted, $t=0.25$ (blue) short dashed dotted line). In panel A we show a comparison between the non interacting and static approximation. The static approximation shows an interesting region of Negative Differential Resistance (NDR), as a consequence of the rich structure of the minima of the generalized potential described in the previous subsection (see also Fig.11 Panel B). At intermediate bias voltage, a strong current currying region appears. This corresponds to $x \sim\left\langle n_{\gamma_{1}}\right\rangle-\left\langle n_{\gamma_{2}}\right\rangle \simeq-0.5$ for which the electronic levels renormalize in the bias window with an effective energy larger than the 'bare' value. Then, for sufficiently large bias, the minimum corresponding to 


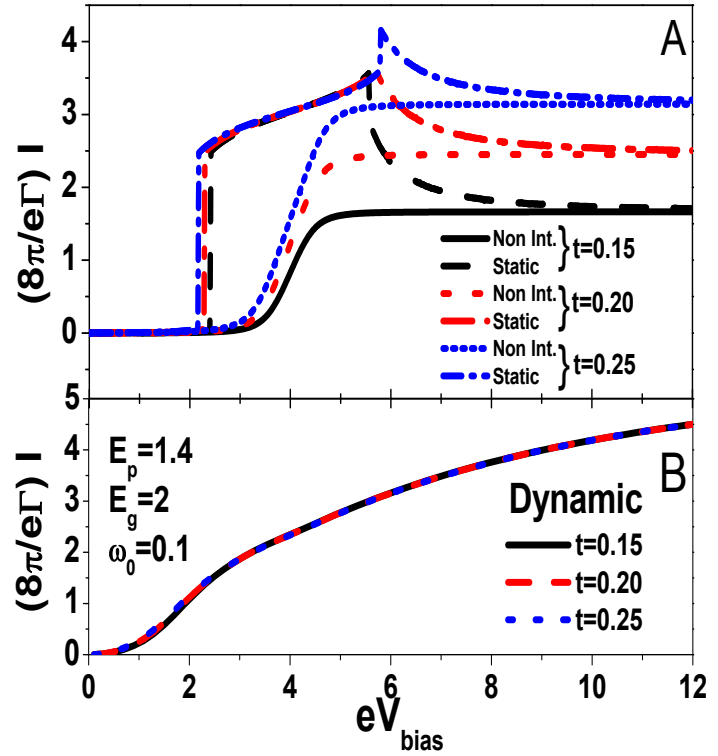

FIG. 16. (Color online). Panel A: Current-Voltage characteristic in the static approximation for $\omega_{0} / \Gamma=0.1, E_{g}=2$ and strong coupling $E_{p}=1.4$ for different values of intermolecular hopping $(t=0.15$ dashed (black), $t=0.2$ dashed dotted (red), $t=0.25$ short dashed dotted (blue)). The non interacting quantities ( $t=0.15$ solid (black), $t=0.2$ dotted (red), $t=0.25$ short dotted (blue)) are also shown. Panel B: Current-Voltage characteristic in the dynamical approximation for the same parameter values of panel $A$. The value of $\omega_{0}$ shown in the figure is in $\Gamma$ units, while all other quantities $\left(E_{g}, E_{p}, t\right.$ and $\left.e V_{\text {bias }}\right)$ are expressed in $\hbar \Gamma$ units.

$x \simeq 0$ prevails, determining a strong current reduction due to the drop of the hoppings to their non interacting 'bare' values. As in the case described above, one can note (panel B) that the dynamical corrections wash out all the features of the static approximation. There is a very small conduction threshold after which one not observe NDR features. Again, we observe that the inclusion of dynamical corrections are very important for a correct description of the SSH model while the static approximation can easily lead to erroneous conclusions.

\section{CONCLUSIONS AND DISCUSSIONS}

In this paper we have derived and studied the stochastic Langevin equation for the dynamics of an oscillator mode coupled to a voltage-biased molecular junction in the adiabatic limit. Using the generalization of the Keldysh formalism to time dependent cases, we were able to show, in agreement with other approaches, that the oscillator dynamics is controlled by an effective potential as well as by damping and fluctuating terms coming from the time depending electronic Green function. Actually, we have built up an expansion for the molecular level Green function in the velocity of the oscillator. In this way we have shown that the quantum effects, 'hidden' in the stochastic equation, come only from the electronic subsystem. Solving numerically the Langevin equation, we have calculated the position and velocity distribution probabilities of the classical oscillator. We have focused our attention on the properties of the velocity distribution function showing that, for sufficiently large bias voltage, it loses its gaussian character. In addition, we have established the range of validity of the adiabatic approximation underlying the stochastic approach by setting QR (small bias), CAR (intermediate bias), CNAR (large bias) regimes. The criterion is based on the comparison of the average kinetic energy of the oscillator with the Debye temperature $\left(k_{B} T_{D} \sim \hbar \omega_{0}\right)$ to distinguish between $\mathrm{QR}$ against CAR regimes, and with electron energy scale $(\sim \hbar \Gamma)$ to distinguish between CAR against CNAR regimes.

We applied our analysis to two simple models of molecules.

For the single site $\mathrm{AH}$ model, the analysis of the validity of the adiabatic approximation has allowed us to build up a diagram for the validity of classical approximation in the plane $\left(E_{p}-V_{\text {bias }}\right)$, showing that the quantum effects are relevant only in a very narrow region if the adiabatic ratio is smaller than all other energy scales. Moreover, we have studied the current-voltage characteristic and the conductance, observing a dynamical reduction of the polaronic shift and the broadening of the electronic resonance due to the average on the nonequilibrium position distribution probability of the oscillator. In the non-gaussian intermediate bias regime and for sufficiently large interaction strength, the kinetic energy shows an interesting non monotonic behaviour. Correspondingly, we observe in the transport properties a strong enhancement of conduction with respect to the infinite mass approximation (static limit).

We have also studied the case of a molecular Hamiltonian composed by a couple of sites interacting with a single vibrational mode in the SSH model. In this case, because of the direct coupling of the electron-oscillator interaction to the intermolecular hopping, the role of the dynamical fluctuations becomes crucial to determine the physical scenario described by the model. The new intermolecular electronic hopping energy scale $t$ introduces a reduction of the CAR in the validity diagram. The new feature is the occurrence of small QRs for sufficiently small coupling $E_{p}$, at intermediate bias voltages. For strong enough electron-phonon coupling $E_{p}$, these regions disappear. In this region of parameters the average dynamical kinetic energy decreases as the bias voltage increases. Also the potential energy curves show this behaviour. Therefore, the oscillator overall energy decreases as a function of bias voltage. This loss of energy occurs for that particular range of bias voltages where the molecular energy levels enter in the bias window. Correspondingly, as in the $\mathrm{AH}$ model case, we observe in the transport properties an enhancement of conduction with respect to the infinite mass approximation. Remarkably, 
in the case of small electron-oscillator interaction and for $E_{g}=0$, we found that the maxima of conductance correspond to the minima of the kinetic energy, shifted by the EOC strength $\alpha$. Finally, within this model, the dynamical corrections on the transport properties cancel out completely the 'detailed' features (like NDR) present in the static case. As main result, we can conclude observing that the inclusion of dynamical effects of the oscillator motion strongly modifies the physical scenario which would be obtained by a static description, even if the oscillator dynamics is much slower than the electron tunneling rate.

We end this section noting that it could be of outstanding interest to study the possibility to include the quantum correction to the oscillator dynamics in the small bias regime classified as Quantum Region (QR). In this direction, Millis et al. ${ }^{40}$ find in the quasi-equilibrium regime $E_{p}>>\omega_{0}>>V_{\text {bias }}$ a quantum contribution to the effective temperature of the oscillator in addition to the diffusive one. At finite mass $m$, nearby the 'gaussian fluctuation' paths involving small excursions (characteristic frequency $\omega_{0}$ ) from the minima of the static potential, quantum tunneling processes become important. The inclusion of the quantum corrections in our approach, within the minimal models considered, is under investigation.

\section{ACKNOWLEDGMENTS}

A.Nocera acknowledges F. Cavaliere and Prof. Sassetti for very useful discussions and CNISM for the financial support.

\section{Appendix A: Abiabatic Approximation}

Here, we show how the adiabatic approximation on the electronic Green function Eq.(9) works.

In order to implement the adiabatic approximation, it is convenient to write the Dyson equation for the molecular retarded Green function (Eq.(9))

$$
\begin{aligned}
G^{r}\left(t, t^{\prime}\right) & =g^{r}\left(t, t^{\prime}\right)+\int d t_{1} \int d t_{2} G^{r}\left(t, t_{1}\right) \\
& +\Sigma_{e l-p h}^{r}\left(t_{1}, t_{2}\right) g^{r}\left(t_{2}, t^{\prime}\right)
\end{aligned}
$$

where the Green function $g^{r}\left(t, t^{\prime}\right)$ already takes into ac- count the coupling with the leads,

$$
g^{r}\left(t, t^{\prime}\right)=-\frac{\imath}{\hbar} \theta\left(t-t^{\prime}\right) e^{-\imath\left(\frac{\varepsilon_{0}}{\hbar}-\imath \Gamma / 2\right)\left(t-t^{\prime}\right)} .
$$

Now, we reparametrize the retarded electron-oscillator self-energy separating slow and fast times scales (in the following, for sake of simplicity, we drop the label $e l-p h$ of the self-energy)

$$
\Sigma^{r}\left(t_{1}, t_{2}\right) \mapsto \Sigma^{r}\left(\frac{t_{1}+t_{2}}{2}, t_{1}-t_{2}\right) .
$$

According to the approach used in Ref.26, we expand Eq. (A3) with respect to the slow mean time $\frac{t_{1}+t_{2}}{2}$ about a generic time $t_{0}$ belonging to the interval $\left[t, t^{\prime}\right]$

$$
\begin{aligned}
& \Sigma^{r}\left(\frac{t_{1}+t_{2}}{2}, t_{1}-t_{2}\right) \simeq \Sigma_{0}^{r}\left(t_{0}, t_{1}-t_{2}\right)+ \\
+ & \Sigma_{1}^{r}\left(t_{0}, \frac{t_{1}+t_{2}}{2}, t_{1}-t_{2}\right),
\end{aligned}
$$

with

$$
\begin{aligned}
& \Sigma_{0}^{r}\left(t_{0}, t_{1}-t_{2}\right)=\lambda x\left(t_{0}\right) \delta\left(t_{1}-t_{2}\right) \\
& \Sigma_{1}^{r}\left(t_{0}, \frac{t_{1}+t_{2}}{2}, t_{1}-t_{2}\right)=\left(\frac{t_{1}+t_{2}}{2}-t_{0}\right) \lambda \dot{x}\left(t_{0}\right) \delta\left(t_{1}-t_{2}\right) .
\end{aligned}
$$

The adiabatic expansion

$$
G^{r}\left(t, t^{\prime}\right) \simeq G_{0}^{r}\left(t_{0}, t-t^{\prime}\right)+G_{1}^{r}\left(t_{0}, t-t^{\prime}\right)
$$

for the Green function follows from that for the selfenergy via the Dyson equation Eq.A1. We can now introduce the Fourier transforms $G_{0 / 1}^{r}\left(t_{0}, \omega\right)=\int d(t-$ $\left.t^{\prime}\right) e^{\imath \omega\left(t-t^{\prime}\right) / \hbar} G_{0 / 1}^{r}\left(t_{0}, t-t^{\prime}\right)$. Since our goal is an adiabatic expansion of the electronic observables at time $t$, we choose $t_{0}=t$. One can easily show that this is the only choice able to recover the fluctuation-dissipation theorem at vanishing bias voltage (equilibrium condition) for the Langevin equation (Eq[17) we derive in Section II.B.1. From the Dyson equation Eq.(A1), taking into account Eq. (A5) and Eq. (A6), we thus find

$$
G^{r}(\omega, t) \simeq G_{0}^{r}(\omega, t)+G_{1}^{r}(\omega, t)
$$

with

$$
\begin{aligned}
G_{0}^{r}(t, \omega) & =\frac{1}{\hbar \omega-E_{g}(t)+\imath \hbar \Gamma / 2}, \\
G_{1}^{r}(t, \omega) & =\imath \hbar \frac{\partial E_{g}}{\partial t} \frac{\partial G_{0}^{r}(t, \omega)}{\partial \hbar \omega} G_{0}^{r}(t, \omega),
\end{aligned}
$$

obtaining a correction which is linear in the velocity of the oscillator $\frac{\partial E_{g}}{\partial t}=\lambda \frac{\partial x}{\partial t}$.
1 Tao N. J., Nat. Nanotechnol. (2006), 1, 173.

2 Reed M.A., Zhou C., Muller C.J., Burgin T.P., Tour J.M., Science 278, 252 (1997)
3 J. Reichert, R. Ochs, D. Beckmann, H.B. Weber, M. Mayor, H.v. Lohneysen, Phys. Rev. Lett. 88, 176804 (2002). 
4 M. Galperin, A. Nitzan and M. A. Ratner, Nano Lett., 5(1), 125 (2005).

5 A. S. Blum, J. G. Kushmerick, et al., Nat. Mater. 4, 167 (2005).

6 M. Blencowe, Contemp. Phys. 46, 249 (2005).

7 M. D. LaHaye, O. Buu, B. Camarota and K. C. Schwab, Science 304, 74 (2004).

8 M. Blencowe and M. Wybourne, Appl. Phys. Lett. 77, 3845 (2000).

${ }^{9}$ R. Knobel and A. N. Cleland, Nature 424, 291 (2003).

10 R. Knobel, Nat. Nano. 3, 525 (2008).

11 T. Holstein, Ann. Phys. (N.Y.) 8, 325 (1959).

12 A. Mitra, I. Aleiner and A. J. Millis, Phys. Rev. B 69, 245302 (2004).

13 M. Galperin, M. A. Ratner, and A. Nitzan, J. Phys.: Condens. Matter 19, 103201 (2007).

14 Wang W., Lee T., Kretzschmar I. and Reed M. A., Nano Lett. 4, 643 (2004).

15 S. Datta, Electronic Transport in Mesoscopic Systems (Cambridge University Press, Cambridge, 1995).

16 L.V. Keldysh, Zh. Eksp. Teor. Fiz. 47, 1515 (1964). [Sov. Phys. JETP 20, 1018 (1965)]

17 H. Haug and A.-P. Jauho, Quantum Kinetics in Transport and Optics of Semiconductors, 2nd ed.,Springer, 2008.

18 D. A. Ryndyk, R. Gutierrez, B. Song and G. Cuniberti, Preprint 0805.0628v1, (2008).

19 J. Koch and F. von Oppen, Phys. Rev. Lett. 94, 206804 (2005).

20 J. Koch, F. von Oppen and A. V. Andreev, Phys. Rev. B 74, 205438 (2006).

21 F. Cavaliere, E. Mariani, R. Leturcq, C. Stampfer and M. Sassetti, Phys. Rev. B 81, 201303 (2010).

22 S. Braig and K. Flensberg, Phys. Rev. B 68, 205324 (2003).
23 D. Mozyrsky, M. B. Hastings and I. Martin, Phys. Rev. B 73, 035104 (2006)

24 F. Pistolesi, Ya. M. Blanter, and I. Martin, Phys. Rev. B 78, 085127 (2008).

25 R. Hussein, A. Metelmann, P. Zedler, and T. Brandes, Phys. Rev. B 82, 165406 (2010).

26 J. Splettstoesser, M. Governale, J. Konig, and R. Fazio, Phys. Rev. Lett. 95, 246803 (2005).

27 F Cavaliere et al., New J. Phys. 10, 115004 (2008).

28 W. P. Su, J. R. Schrieffer, and A. J. Heeger, Phys. Rev. Lett. 42, 1698 (1979).

29 A. J. Heeger, S. Kivelson, and J. R. Schrieffer, Rev. Mod. Phys. 60, 781 (1988).

${ }^{30}$ H. Ness, S. A. Shevlin, and A. J. Fisher, Phys. Rev. B 63, 125422 (2001).

31 Y. Asai, Phys. Rev. B 78, 045434 (2008).

32 G. A. Kaat and K. Flensberg, Phys. Rev. B 71, 155408 (2005).

33 R. Messina and R. Passante, Phys. Rev. A 76, 032107 (2007).

34 W. T. Coffey, Yu. P. Kalmykov, and J. T. Waldron, The Langevin Equation, 2nd ed. (World Scientific, Singapore, 2004); N. G. Van Kampen, Stochastic Processes in Physics and Chemistry (North-Holland, Amsterdam, 1981); H. Risken, The Fokker-Planck equation. Methods of solution and applications (Springer-Verlag, New York, 1984).

35 R. L. Honeycutt, Phys. Rev. A 45, 600 (1992).

36 R.L. Honeycutt, Phys. Rev. A 45, 604-610 (1992).

37 J. D. Bao, R. W. Li, W. Wu, J. Comput. Phys. 197, 241 (2004).

38 A. N. Pasupathy et al., Nano Lett. 5, 203 (2005).

39 Y. Meir and N.S. Wingreen, Phys. Rev. Lett. 68, 2512 (1992)

40 A. Mitra, I. Aleiner and A. J. Millis, Phys. Rev. Lett. 94, 076404 (2005). 